\title{
Time-Inconsistent Portfolio Investment Problems
}

\author{
Yidong Dong* $\quad$ Ronnie Sircar ${ }^{\dagger}$
}

April 2014; revised October 6, 2014

\begin{abstract}
The explicit results for the classical Merton optimal investment/consumption problem rely on the use of constant risk aversion parameters and exponential discounting. However, many studies have suggested that individual investors can have different risk aversions over time, and they discount future rewards less rapidly than exponentially. While state-dependent risk aversions and non-exponential type (e.g. hyperbolic) discounting align more with the real life behavior and household consumption data, they have tractability issues and make the problem time-inconsistent. We analyze the cases where these problems can be closely approximated by time-consistent ones. By asymptotic approximations, we are able to characterize the equilibrium strategies explicitly in terms of the corrections to solutions for the base problems with constant risk aversion and exponential discounting. We also explore the effects of hyperbolic discounting under proportional transaction costs.
\end{abstract}

\section{Introduction and Background}

\subsection{The Merton Problem of Portfolio Optimization}

The portfolio optimization problem in a continuous-time diffusion model was first introduced by Merton in the 1960s, with the original papers reprinted later in [24], where he was able to derive explicit solutions for the value functions and optimal strategies in cases with geometric Brownian motions and special types of utility functions. Ever since then, there has been plenty of development aimed at generalizing Merton's results in different ways. To deal with market incompleteness is a direction that a large proportion of the works have been dedicated to. For example, Campbell and Viceira [4] and Wachter [31] studied the problem with stochastic drift returns. For problems of partial hedging with a non-traded asset, as well as utility indifference pricing, one could refer to the collection [5]. Meanwhile, stochastic volatility and transaction cost are two topics that have received much attention and popularity. We refer the reader to Chacko and Viceira [6] and Kraft [22] for some explicit results in cases with stochastic volatility. For transaction costs, things are much more subtle as the problem becomes less tractable. Davis and Norman [10] were able to solve the problem numerically as a free-boundary ODE system, and Shreve and Soner [28] treated it using the viscosity solution approach.

More recently, asymptotic methods have been used widely to solve the extensions of Merton's problem around their classical and well-established counterpart problems. For example, Fouque et al. [15] have

\footnotetext{
*Department of Operations Research and Financial Engineering, Princeton University, Princeton, NJ 08544, USA. yidongdeprinceton.edu.

${ }^{\dagger}$ Department of Operations Research and Financial Engineering, Princeton University, Princeton, NJ 08544, USA. sircareprinceton. edu. Partially supported by NSF grant DMS-1211906.
} 
used multiscale expansions to approximate the case with stochastic volatility around the constant volatility case. Bouchard et al. [3] used asymptotics for small transaction costs to derive tractable models for a partial hedging problem under expected loss constraints.

\subsection{Time Inconsistency}

The key to solving Merton's problem is the use of the Dynamic Programming Principle (hereinafter DPP) in order to formulate the Hamilton-Jacobi-Bellman (hereinafter HJB) equation. In a typical dynamic programming problem setup, when an agent wants to optimize an objective function by choosing the best plan, he is only required to decide his current action. This is because DPP assumes that one's future selves are going to solve the remaining part of today's problem and act optimally when future comes. However, in many problems, the DPP does not hold, and an agent does not have such "commitment power" on their future selves, which is the ability to enforce a course of plans obtained by repeatedly optimizing the same objective function over time. In such problems, the future selves may have changed preferences or tastes, or would want to make decisions based on different objective functions, effectively acting as opponents of the current self.

The dilemma described above is called dynamic inconsistency, which has been noted and studied by economists for many years, mainly in the context of non-exponential type discount functions. In [29], Strotz demonstrated that when a discount function was applied to consumption plans, one could favor a certain plan at the beginning, but later switch preference to another plan. This would hold true for most types of discount functions, the only exception being the exponential. Nevertheless, exponential discounting is the default setting in most literatures, as none of the other types could produce explicit solutions. Results from experimental studies contradict this assumption (see, for example, Loewenstein and Prelec [23]), indicating that the discount rates for the near future are much lower than the discount rates for the time further away in future, and therefore a hyperbolic type discount function would be more realistic.

Other types of time-inconsistency do exist as well. Bjork and Murgoci [1] listed out three possible scenarios where time inconsistency would occur in typical Markovian stochastic control problems. More specifically, given an objective function of the following form:

$$
J(t, x, \pi)=\mathbb{E}\left[\int_{t}^{T} \varphi(s-t) F\left(X_{s}^{\pi}, x\right) d s+G\left(X_{T}^{\pi}, x\right)\right]+H\left(x, \mathbb{E}\left[X_{T}^{\pi}\right]\right),
$$

where $X^{\pi}$ is some controlled diffusion process with $X_{t}^{\pi}=x$ and $\pi$ being our control, the optimization for $J(t, x, \pi)$ is a time-inconsistent problem if:

1. the discount function $\varphi(s-t)$ is not of exponential type, e.g. a hyperbolic discount function;

2. $x$ appears in the objective function, e.g. a utility function that depends on the initial wealth $x$;

3. $H()$ is a nonlinear function of $\mathbb{E}\left[X_{T}^{\pi}\right]$, e.g. continuous-time mean-variance optimization on $X_{T}^{\pi}$.

In all the three cases, the standard HJB equations cannot be derived since the usual formulation requires an argument about the value function (process) being a supermartingale for arbitrary controls and being a martingale at optimum, which does not hold here. In light of the non-applicability of DPP on these problems, some have turned in a game-theoretic direction. By treating the problem as a game played with one's future selves, it is possible to derive a sub-game Nash equilibrium. In the next section, we will discuss recent work on deriving equilibrium strategies in some of the time-inconsistent problems. 


\subsection{Recent Literature on Time-inconsistent Portfolio Optimization}

One of the earlier advances was made by Harris and Laibson who discussed the existence and uniqueness of an equilibrium consumption path in the case of hyperbolic (in [16]) and quasi-hyperbolic (in [17]) discount functions in a discrete-time setup. They also derived the Euler relation for the equilibrium path using the recursive property of an equilibrium consumption plan. Ekeland and Lazrak [11] studied the problem in continuous time with a more general non-exponential type discount function and derived an equation for the equilibrium value function process, which was comparable to Harris and Laibson's results and resembles the classical HJB equation plus a non-local term. Later, Ekeland et al. [13] looked at an investment/consumption problem from life insurance with time-inconsistent discount functions. They solved the non-local HJBtype equation numerically and were able to obtain a hump-shaped consumption path that agreed with the household consumption data, as opposed to the monotone shape path produced by exponential discount functions.

Progress has been made on other types of time inconsistent problems as well. Bjork et al. [2] used the same technique to study the continuous time mean-variance optimization problem with a state-dependent risk aversion parameter. They obtained a system of HJB-type equations which they were able to reduce to an ODE system and solve numerically if risk aversion had a special form. Their equilibrium strategy was comparable to the utility-maximizing strategy in a Merton model statically, but was able to capture some horizon effect as opposed to the Merton optimal strategy which was constant in time. On the other hand, Hu et al. [18] derived an open-loop equilibrium strategy, characterized by a system of forward-backward stochastic differential equations, to solve a time-inconsistent stochastic linear quadratic control problem, which is the generalized version of the mean-variance problem. Pirvu and Zhang [26] have studied the problem of utility indifference pricing under a discrete time model with a state-dependent risk aversion modeled by a two-state regime-switching Markov chain.

The remaining part of this article is organized as follows. In Section 2, we study the portfolio optimization problem with time-varying risk aversions that depend on the wealth or volatility factor. A discrete-time example will be given to illustrate the time inconsistency, followed by the derivation of the HJB-type equation in continuous time. We will use asymptotic methods to derive the equilibrium strategies up to first order. In Section 3, we look at hyperbolic discounting problems and use similar methodologies to obtain tractable solutions in this case. An extension with proportional transaction costs is also studied, and we provide some numerical results for this problem. Section 4 concludes.

\section{Utility Maximization with Time-varying Risk Aversion}

In this section, we look at the classical Merton's problem of portfolio optimization, but with the risk aversion parameter being state-dependent ${ }^{1}$. Our motivation is that, in the classical case, we need to, at time 0 , fix a (constant) risk aversion parameter for expected utility at terminal time $T$. This value reflects our present conjecture about our future attitude towards risk, and thus it would be unnatural for this conjecture to be independent of the current state of the world, for example the wealth level and economic conditions. There are many indicators in the market that can, at least partially, measure investors' risk aversion. As mentioned in Coudert and Gex [9], the movement of risk aversion is often correlated with market indices, for example the gold price and VIX. There are also aggregate indicators of risk aversion created by financial institutions such as JP Morgan's Liquidity, Credit and Volatility Index. The consequence of incorporating

\footnotetext{
${ }^{1}$ These models can be seen as a particular example of the studies on state-dependent utility/preference by Karni [20]. In this case the dependency has an explicit functional form as $\gamma(\cdot)$.
} 
such dependence is that the problem now becomes time-inconsistent, as the risk aversion will likely to be different at a later time leading to a different objective function to optimize. An example is provided in the next section as an illustration. We will follow closely the methodology described in [1]. As we will see later, a system of equations of the HJB type can be obtained in this manner, which admits the equilibrium solution via first order conditions. And when the risk aversion is constant, this system will degenerate to the classical HJB equation.

\subsection{Time-inconsistency and Wealth-dependent Risk Aversion}

To keep the dimension small, we start by describing the time-inconsistency problem with the risk aversion being dependent on the current wealth level, $X_{t}$. Since the current wealth level is an indicator on how much loss (downside risk) one is able to bear, we believe this dependence is natural. We will illustrate the derivation of the HJB-type system of equations in this case, which can be easily extended to cases where risk aversion depends on other state variables.

\subsubsection{An Illustration}

We can use a simple two-period binomial tree to illustrate the time inconsistency that results from the wealthdependent risk aversion. Let $k \in\{0,1,2\}$ denote the time periods. Suppose there are two assets $S_{k}$ and $B_{k}, B_{k}$ being the risk free asset and $S_{k}$ being the risky one with $u>1, d<1$ and $p \in[0,1]$ as the usual parameters in a binomial tree model. We also assume that both assets have value equal to 1 at time 0 and we have zero interest rate so $B_{k}=1 \forall k$.

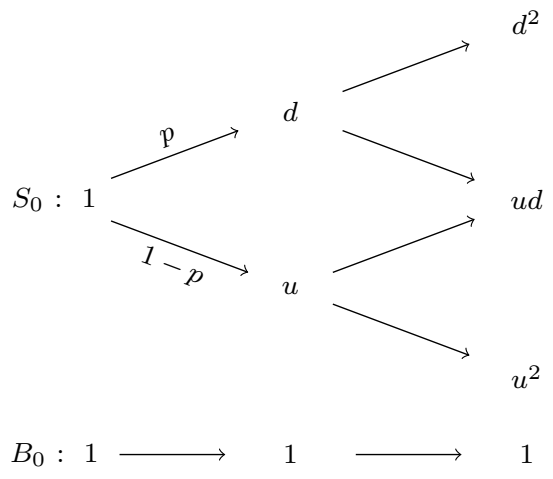

Let $X_{k}$ denote our wealth at time $k$ and suppose $X_{0}=1$ for simplicity. We use an exponential utility function $U(x)=-e^{-\gamma x}$ here, and we let the risk aversion $\gamma$ be a function of the current wealth level (denoted as $x$ here):

$$
\gamma(x)=\left\{\begin{array}{lll}
a & \text { if } & x>1 \\
1 & \text { if } & x=1 \\
b & \text { if } & x<1
\end{array}\right.
$$

for $a, b>0$.

Let $0<\pi<1$ denote the amount of wealth invested in $S_{k}$ at time 0 . At time $k=0$, with wealth $X_{0}=1$, the expected utility of terminal wealth $X_{2}^{\pi}:=\pi S_{2}+(1-\pi) B_{2}$ can be written as:

$$
\begin{aligned}
\mathbb{E}\left[U\left(X_{2}^{\pi}\right)\right] & =-e^{-1}\left\{p^{2} e^{\pi\left(1-u^{2}\right)}+2 p(1-p) e^{\pi(1-u d)}+(1-p)^{2} e^{\pi\left(1-d^{2}\right)}\right\} \\
& =:-e^{-1} f_{1}(\pi)
\end{aligned}
$$


where the risk aversion $\gamma=1$. At time $k=1$, depending on whether the stock price goes up or down, the risk aversion will becomes $\gamma=a$ or $b$ because we have either $X_{1}>1$ or $X_{1}<1$. The expected utility of $X_{2}^{\pi}$ at time 1 is either

$$
\mathbb{E}\left[U\left(X_{2}^{\pi}\right) \mid S_{1}=u\right]=-e^{-1}\left\{p e^{\pi\left(1-u^{2}\right) a}+(1-p) e^{\pi(1-u d) a}\right\}=:-e^{-1} f_{2}(\pi),
$$

or

$$
\mathbb{E}\left[U\left(X_{2}^{\pi}\right) \mid S_{1}=d\right]=-e^{-1}\left\{p e^{\pi(1-u d) b}+(1-p) e^{\pi\left(1-d^{2}\right) b}\right\}=:-e^{-1} f_{3}(\pi) .
$$

Remark 2.1. It is possible to choose $p, u, d, \pi$ such that

$$
\frac{\partial}{\partial \pi} f_{1}(\pi)>0, \quad \frac{\partial}{\partial \pi} f_{2}(\pi)<0 \quad \text { and } \quad \frac{\partial}{\partial \pi} f_{3}(\pi)<0 .
$$

For instance, if $a=0.5$ and $b=2$, then by choosing $u=2, d=0.5, p=0.5$ and $\pi=0.5$ we can obtain the desired inequalities.

Suppose we have Portfolio \#1 that has $\pi$ in the stock and $1-\pi$ in the bank, and Portfolio \#2 that has $\pi-\epsilon$ in stock and $1-\pi+\epsilon$ in the bank for an infinitesimal positive amount $\epsilon$. The signs of the first derivatives in Remark 2.1 tell us that, at the second period, Portfolio \#1 is always favored over Portfolio \#2. However, at time 0, Portfolio \#2 is the better one. We recall the definition of time consistent utility function, such as in [21] and [7]:

Definition 2.2. A dynamic utility function $\left(U_{t}\right)_{t=0}^{T}$ is time-consistent if for all $X, Y \in L\left(\mathcal{F}_{T}\right)$ and $t \in$ $0, \ldots, T-1$,

$$
U_{t+1}(X) \geq U_{t+1}(Y) \text { implies } U_{t}(X) \geq U_{t}(Y)
$$

We can see that in our case the preference between Portfolio \#1 and \#2 is "flipped" in the two periods, which clearly violates the definition of time consistency.

\subsubsection{Formal Problem Setup in Continuous Time}

We use the standard two-asset framework where we have a risky stock $S_{t}$ and a risk-free bond that we can invest our wealth in. By assuming zero interest rate or working under the discounted unit, we only need to define the stock dynamics:

$$
d S_{t}=\mu S_{t} d t+\sigma S_{t} d W_{t}
$$

where $W_{t}$ denotes a standard Brownian motion, so $S_{t}$ is a geometric Brownian motion (henceforth GBM). For the time being, we assume constant drift $\mu$ and volatility $\sigma$. The case where the volatility is stochastic will be discussed in Section 2.3 when we consider the volatility-dependent risk aversion as an extension of this problem.

Let $X_{t}$ denote our wealth at time $t$, which consists of the cash amount $\pi_{t}$ invested in the risky stock as well as the remaining part invested in the riskless bond. The wealth process $X_{t}$ follows the controlled diffusion:

$$
d X_{t}=\pi_{t} \frac{d S_{t}}{S_{t}}=\pi_{t}\left[\mu d t+\sigma d W_{t}\right]
$$


The optimization problem is to maximize the expected utility of terminal wealth at time $T$ among all admissible strategies $\pi$, given the wealth level being $X_{t}=x$ at time $t$. This problem can be represented using the value function $V(t, x)$

$$
\begin{aligned}
V(t, x) & :=\sup _{\boldsymbol{\pi} \in \boldsymbol{\Pi}} \mathbb{E}_{t, x}\left[U\left(X_{T}^{\boldsymbol{\pi}}, \gamma\left(X_{t}\right)\right)\right] \\
& =\sup _{\boldsymbol{\pi} \in \boldsymbol{\Pi}} \mathrm{J}(t, x, \boldsymbol{\pi}),
\end{aligned}
$$

where $\Pi$ is the set of all admissible strategies that are adapted to the filtration $\left(\mathcal{F}_{s}\right)$ generated by the stock price process and which satisfy $\mathbb{E}\left[\int_{t}^{T} \pi_{s}^{2} d s<\infty\right]$, and $\gamma\left(X_{t}\right)$ is the risk aversion that we fix for our future self at time $T$ based on the current wealth level $X_{t}$. Here $U\left(x_{1}, \gamma\left(x_{2}\right)\right)$ denotes the von NeumannMorgenstern utility function which is a twice differentiable, concave function in $x_{1} \in \mathbb{R}^{+}$.

Remark 2.3. Note that in $U\left(x_{1}, \gamma\left(x_{2}\right)\right), x_{1}$ is the wealth at future for which we want to compute the utility, with risk aversion computed using current wealth level $x_{2}$. In order to retain the differentiability and concavity at terminal time $T$ when $x_{1}$ and $x_{2}$ coincide, we also require $\gamma\left(x_{2}\right)$ to be chosen such that

- $U\left(x_{1}, \gamma\left(x_{2}\right)\right)$ is twice differentiable in $x_{2}$;

- $\left.\left(U_{x_{1} x_{1}}+U_{x_{2} x_{2}}+2 U_{x_{1} x_{2}}\right)\right|_{x_{2}=x_{1}}<0$ for all $x_{1}>0$.

In the classical case, the risk aversion $\gamma$ is constant so we can suppress its argument by denoting the utility function as $U=U^{\gamma}(x)$. Then the optimal strategy can be computed from the HJB equation associated with the value function, and the DPP, from which the HJB equation is derived, guarantees that the optimal strategy $\pi^{*}$ computed at the initial time will remain optimal at a later time. A rigorous proof of the derivation of HJB equation from DPP can be found in, for example, Pham [25]. In this case, the optimal strategy takes the form:

$$
\pi_{t}^{*}=\left\{\begin{array}{l}
\frac{\lambda}{\sigma} \frac{1}{\gamma} \text { for exponential utility functions } \\
\frac{\lambda}{\sigma} \frac{x}{\gamma} \text { for log and power utility functions, }
\end{array}\right.
$$

where $X_{t}=x$ and $\lambda$ is the Sharpe ratio defined by $\lambda:=\frac{\mu}{\sigma}$.

Now, as we have made the risk aversion wealth dependent, intuitively the optimal strategy might be obtained by replacing the constant $\gamma$ with $\gamma(x)$ in the above expressions. Is it really the case? It turns out that this is not so trivial, since we cannot even formulate the HJB equation (in the classical DPP sense) once we allow the risk aversion to change with the current wealth level. As our objective function changes constantly, our future selves will not solve the "remaining" part of the optimization problem that our current self is facing now.

Using a game-theoretic approach, we can think of it as a game played by a number of ordered players (our selves at different times), each of whom has his own utility function and has temporary control over the resource (wealth). For a particular player, when the resource is in his possession (obtained from the previous player), he can choose the strategy to be applied to the resource at this particular moment. After that, the player has to pass on the resource to the next player and he will no longer be able to apply strategies to it or control what other players' strategies will be. As the game is played by a continuum of players in the continuous time setting, each player would have to play against all his future selves.

To define the equilibrium in this game, we follow the explanation given in [11], in which it was assumed that the current self has the ability to commit all future selves to his decision up to a small period $\epsilon>0$. 
Thus the player can form a small coalition with players in the near future. Now suppose $\boldsymbol{\pi} \equiv\left(\pi_{s}\right)_{s \in[t, T]}$ is an admissible policy (all strategies over time). Define another policy $\boldsymbol{\pi}_{\epsilon}$ as:

$$
\boldsymbol{\pi}_{\epsilon}=\left\{\begin{array}{l}
\pi, \quad s \in[t, t+\epsilon] \\
\pi_{s}, \quad s \in(t+\epsilon, T]
\end{array}\right.
$$

where $\pi$ can be any strategy that makes $\pi_{\epsilon}$ admissible. Then the following from [11] gives the definition of the equilibrium policy.

Definition 2.4. A policy $\overline{\boldsymbol{\pi}}:(t, x) \rightarrow \mathbb{R}$ is an equilibrium one if for any $t, x>0$ and any arbitrary $\pi$,

$$
\lim _{\epsilon \downarrow 0} \frac{\mathrm{J}(t, x, \overline{\boldsymbol{\pi}})-\mathrm{J}\left(t, x, \overline{\boldsymbol{\pi}}_{\epsilon}\right)}{\epsilon} \geq 0
$$

where $\mathrm{J}$ is our objective function.

This definition means that, if we are using the equilibrium policy $\overline{\boldsymbol{\pi}}$, then we will not be better off by committing the immediate future selves to our action instead of letting them choose the best strategy in their views. This also means that the equilibrium policy computed at one time should coincide, from the next period onward, with the equilibrium policy computed at the next period. The equilibrium policy is therefore time-consistent as the future selves have no incentives to deviate from this path. We refer the readers to the paragraphs following Definition 1 in Ekeland and Lazrak [12] for a detailed explanation about the equilibrium strategy in discrete time setting. The definition leads to the following result as appeared in, e.g. [1]:

Proposition 1. Assuming sufficient regularity, the equilibrium value function and Markovian policy satisfy the following extended HJB-type system:

$$
\left\{\begin{aligned}
\sup _{\pi_{t} \in \mathbb{R}}\left(\mathcal{A}^{\pi_{t}} V(t, x)-\mathcal{A}^{\pi_{t}} f(t, x, x)+\mathcal{A}^{\pi_{t}} f^{w}(t, x)\right) & =0 \\
\mathcal{A}^{\pi_{t}^{*}} f^{w}(t, x) & =0 \\
V(T, x) & =U(x, \gamma(x)) \\
f(T, x, w) & =U(x, \gamma(w)),
\end{aligned}\right.
$$

where $\mathcal{A}^{\pi_{t}}$ contains the infinitesimal generator of the wealth process taking the form:

$$
\begin{aligned}
\mathcal{A}^{\pi_{t}} g(t, x) & =g_{t}+\mu \pi_{t} g_{x}+\frac{1}{2} \sigma^{2} \pi_{t}^{2} g_{x x} \\
\mathcal{A}^{\pi_{t}} h(t, x, x) & =h_{t}+\mu \pi_{t} h_{x}+\mu \pi_{t} h_{w \mid w=x}+\frac{1}{2} \sigma^{2} \pi_{t}^{2} h_{x x}+\frac{1}{2} \sigma^{2} \pi_{t}^{2} h_{w w \mid w=x}+\sigma^{2} \pi_{t}^{2} h_{x w \mid w=x},
\end{aligned}
$$

and $f^{w}(t, x)$ means fixing the $w$ variable of $f(t, x, w)$ as constant.

Proof. We need to define the following "auxiliary value function":

$$
f(t, x, w)=\mathbb{E}_{t, x}\left[U\left(X_{T}^{\boldsymbol{\pi}^{*}}, \gamma(w)\right)\right],
$$

which is made from $V(t, x)$ by making the initial wealth value in $\gamma(\cdot)$ vary independently from $X_{t}$, and where $\boldsymbol{\pi}^{*}$ denotes the equilibrium strategy. For every fixed $w, \gamma(w)$ can be treated as a constant, as $w$ and 
$x$ are independent. Thus $f(t, x, w)$ is the value function for a Merton problem with constant risk aversion parameter $\gamma(w)$.

By construction of $\boldsymbol{\pi}_{\epsilon}$ from the definition, we have the following equality:

$$
\begin{aligned}
\mathbb{E}_{t, x}\left[\mathrm{~J}\left(t+\epsilon, X_{t+\epsilon}, \boldsymbol{\pi}_{\epsilon}\right)\right] & =\mathbb{E}_{t, x}\left[f\left(t+\epsilon, X_{t+\epsilon}, X_{t+\epsilon}\right)\right] \\
& =\mathbb{E}_{t, x}\left[f\left(t+\epsilon, X_{t+\epsilon}, X_{t+\epsilon}\right)\right]+\mathrm{J}(t, x, \boldsymbol{\pi})-\mathbb{E}_{t, x}\left[U\left(X_{T}^{\boldsymbol{\pi}}, \gamma(x)\right)\right] \\
& =\mathbb{E}_{t, x}\left[f\left(t+\epsilon, X_{t+\epsilon}, X_{t+\epsilon}\right)\right]+\mathrm{J}(t, x, \boldsymbol{\pi})-\mathbb{E}_{t, x}\left[\mathbb{E}\left[U\left(X_{T}^{\boldsymbol{\pi}}, \gamma(x)\right) \mid X_{t+\epsilon}, t\right]\right] \\
& =\mathbb{E}_{t, x}\left[f\left(t+\epsilon, X_{t+\epsilon}, X_{t+\epsilon}\right)\right]+\mathrm{J}(t, x, \boldsymbol{\pi})-\mathbb{E}_{t, x}\left[f\left(t+\epsilon, X_{t+\epsilon}, x\right)\right] .
\end{aligned}
$$

Since $\mathrm{J}\left(t+\epsilon, X_{t+\epsilon}, \boldsymbol{\pi}_{\epsilon}\right)=V\left(t+\epsilon, X_{t+\epsilon}\right)$, we can write the above equation as:

$$
\mathbb{E}_{t, x}\left[V\left(t+\epsilon, X_{t+\epsilon}\right)\right]=\mathbb{E}_{t, x}\left[f\left(t+\epsilon, X_{t+\epsilon}, X_{t+\epsilon}\right)\right]+\mathrm{J}(t, x, \boldsymbol{\pi})-\mathbb{E}_{t, x}\left[f\left(t+\epsilon, X_{t+\epsilon}, x\right)\right] .
$$

Taking the supremum and rearranging the equation, we get

$$
\sup _{\boldsymbol{\pi} \in \boldsymbol{\Pi}}\left(\mathbb{E}_{t, x}\left[V\left(t+\epsilon, X_{t+\epsilon}\right)\right]-V(t, x)+\mathbb{E}_{t, x}\left[f\left(t+\epsilon, X_{t+\epsilon}, x\right)\right]-\mathbb{E}_{t, x}\left[f\left(t+\epsilon, X_{t+\epsilon}, X_{t+\epsilon}\right)\right]\right)=0 .
$$

Now we take the limit $\epsilon \rightarrow 0$,

$$
\sup _{\pi_{t}}\left(\mathcal{A}^{\pi_{t}} V(t, x)-\mathcal{A}^{\pi_{t}} f(t, x, x)+\mathcal{A}^{\pi_{t}} f^{w \mid w=x}(t, x)\right)=0 .
$$

Meanwhile, for every fixed $w, f\left(t, X_{t}, w\right)$ corresponds to a martingale process and thus it must satisfy the PDE

$$
A^{\pi_{t}^{*}} f^{w}(t, x)=0
$$

where $\pi^{*}$ is the equilibrium policy appeared in the definition of $f(t, x, w)$. In addition, there are two terminal conditions for $V$ and $f$ :

$$
\left\{\begin{aligned}
V(T, x) & =U(x, \gamma(x)) \\
f(T, x, w) & =U(x, \gamma(w)) .
\end{aligned}\right.
$$

We get the extended HJB-type system by combining equations (6), (7) and (8).

The verification theorem provided by [1] holds here, we shall quote:

Theorem 1 (Bjork and Murgoci). Assume that $(V(t, x), f(t, x, w))$ is a solution of the system defined in (5), and that the strategy path $\pi^{*}$ realizes the supremum in the equation. Then $\pi^{*}$ is an equilibrium policy, and $V(t, x)$ is the corresponding value function.

Proof. See [1].

When writing out the first two equations in (5) explicitly, we get the following two PDEs:

$$
\left\{\begin{aligned}
V_{t}+\sup _{\pi}\left\{\mu \pi\left(V_{x}-f_{w}\right)+\frac{1}{2} \sigma^{2} \pi^{2}\left(V_{x x}-f_{w w}-2 f_{x w}\right)\right\} & =0 \\
f_{t}+\mu \pi^{*} f_{x}+\frac{1}{2} \sigma^{2} \pi^{* 2} f_{x x} & =0 .
\end{aligned}\right.
$$

Note that all $w$ partial derivatives are evaluated at the point $w=x$. 
We can find the equilibrium strategy by the first order condition:

$$
\pi^{*}=-\left.\frac{\lambda}{\sigma} \frac{V_{x}-f_{w}}{V_{x x}-f_{w w}-2 f_{x w}}\right|_{w=x},
$$

where $\lambda$ denotes the constant Sharpe ratio. Inserting (10) back into (9) and we obtain the following two PDEs to solve for $V(t, x)$ and $f(t, x, w)$

$$
\left\{\begin{aligned}
V_{t}-\frac{1}{2} \lambda^{2} \frac{V_{x}^{2}-2 V_{x} f_{y}+f_{w}^{2}}{V_{x x}-f_{w w}-2 f_{x w}} & =0 \\
f_{t}+\lambda^{2}\left[\frac{f_{x}\left(f_{w}-V_{x}\right)}{V_{x x}-f_{w w}-2 f_{x w}}+\frac{1}{2} \frac{\left(V_{x}^{2}-2 V_{x} f_{w}+f_{w}^{2}\right) f_{x x}}{\left(V_{x x}-f_{w w}-2 f_{x w}\right)^{2}}\right] & =0 .
\end{aligned}\right.
$$

\subsubsection{A Remark: Why Two Equations Instead of One?}

As we can see from above, we now face an HJB-type system of two equations instead of solving one single HJB equation as in the time-consistent case. It turns out this is essential for characterizing the equilibrium strategy and value function. In the definition of the equilibrium strategy, coalition is allowed for an infinitesimal period, during which we are actually solving a Merton problem with constant risk aversion. That is what the function $f(t, x, w)$ represents when setting $w=x$ and it is the time-consistent part of the problem. After this infinitesimal period, however, the evolution of the value function cannot be characterized by this function $f(t, x, w)$ any more, as the problem now is time-inconsistent. This is the reason we need $V(t, x)$ as our value function.

In Harris and Laibson [17], the dynamic consumption choice problem with quasi-hyperbolic discounting was also solved by the equilibrium strategy and value function that were defined similarly using two functions. There is a continuation-value function characterizing the dynamics of the true time-inconsistent value function and there is another current-value function, which is used locally at the current point $(t, x)$ to derive the equilibrium strategy. Our HJB-type system has a strong analogy to the two functions in their work.

Another way of describing this is that the true value function $V(t, x)$ cannot be solved alone. For each point $(t, x)$, the value of $V(t, x)$ is determined by another non-local function $f(t, x, w)$ by setting $w=x$ in its third argument, i.e. $V(t, x)=f(t, x, x)$. The first equation in (5) can be considered as a PDE of the non-local type. For the non-exponential discounting problem in [11], a non-local integro-PDE was obtained, where the dynamics of the value function depends on an integral of the value function at all future time. In general, non-local PDEs are very difficult to solve.

\subsubsection{Asymptotic Expansions}

If the time-inconsistent problem is close to a time-consistent one, we can approximate the first problem very effectively using the latter by asymptotic methods. Here we look at the special case where the risk aversion only varies slowly with the wealth level, i.e. it is close to the case of constant risk aversion. Mathematically, this corresponds to

$$
\gamma(x)=\gamma_{0}+\epsilon \gamma_{1}(x)+\cdots
$$

for positive $\epsilon \ll 1$. We look for an expansion of the form

$$
V(t, x)=V_{0}(t, x)+\epsilon V_{1}(t, x)+\cdots,
$$


and

$$
f(t, x, w)=f_{0}(t, x)+\epsilon f_{1}(t, x, w)+\cdots,
$$

for the equations in (11).

We first introduce a few notations.

Definition 2.5. We define the risk tolerance to be

$$
R:=-\frac{V_{0, x}}{V_{0, x x}}
$$

and we use $\mathcal{D}_{k}$ to denote

$$
\mathcal{D}_{k}:=R^{k} \frac{\partial^{k}}{\partial x^{k}}
$$

and finally define the linear operator $\mathcal{L}_{t, x}$ as

$$
\mathcal{L}_{t, x}:=\partial_{t}+\lambda^{2} \mathcal{D}_{1}+\frac{1}{2} \lambda^{2} \mathcal{D}_{2}
$$

Collecting zeroth order terms in (11) we get

$$
\left\{\begin{array}{l}
\mathcal{L}_{t, x} V_{0}=0 \\
\mathcal{L}_{t, x} f_{0}=0
\end{array}\right.
$$

with terminal conditions $V_{0}(T, x)=U\left(x, \gamma_{0}\right)$ and $f_{0}(T, x)=U\left(x, \gamma_{0}\right)$. Since $f_{0}$ and $V_{0}$ have the same terminal condition, we find $V_{0}(t, x)=f_{0}(t, x)$ which is the classical Merton value function with constant risk aversion parameter $\gamma_{0}$.

At the first order, we have:

$$
\left\{\begin{array}{l}
\mathcal{L}_{t, x} V_{1}=\lambda^{2} R f_{1, w}+\frac{\lambda^{2}}{2} R^{2}\left(f_{1, w w}+2 f_{1, x w}\right) \\
\mathcal{L}_{t, x} f_{1}=0
\end{array}\right.
$$

with terminal conditions $V_{1}(T, x)=\frac{\partial U}{\partial \gamma}\left(x, \gamma_{0}\right) \gamma_{1}(x)$ and $f_{1}(T, x, w)=\frac{\partial U}{\partial \gamma}\left(x, \gamma_{0}\right) \gamma_{1}(w)$. The following proposition will be useful for solving the order $\epsilon$ PDEs.

Lemma 1. We have

$$
\frac{\partial}{\partial \gamma} \mathcal{L}_{t, x} V_{0}=\mathcal{L}_{t, x}\left(\frac{\partial V_{0}}{\partial \gamma}\right)
$$

Proof. For any function $v$,

$$
\begin{aligned}
\frac{\partial}{\partial \gamma} \mathcal{L}_{t, x} v & =\frac{\partial}{\partial \gamma}\left(v_{t}+\lambda^{2} R v_{x}+\frac{1}{2} \lambda^{2} R^{2} v_{x x}\right) \\
& =\mathcal{L}_{t, x}\left(\frac{\partial v}{\partial \gamma}\right)+\lambda^{2} \frac{\partial R}{\partial \gamma} v_{x}+\lambda^{2}\left(R \frac{\partial R}{\partial \gamma}\right) v_{x x}
\end{aligned}
$$

The last two terms cancel out when $v=V_{0}$ since $R=-\frac{V_{0, x}}{V_{0, x x}}$. 
Lemma 1 will lead us to the solutions of $f_{1}$ and $V_{1}$.

Proposition 2. The solution to the second equation in (13) is

$$
f_{1}(t, x, w)=\gamma_{1}(w) \frac{\partial f_{0}}{\partial \gamma} .
$$

Therefore the order $\epsilon$ value function is

$$
V_{1}(t, x)=\gamma_{1}(x) \frac{\partial V_{0}}{\partial \gamma}
$$

Proof. By direct substitution and verification.

\subsubsection{Effect on the Trading Strategy}

Recall the equilibrium strategy from (10)

$$
\pi^{*}=-\frac{\lambda}{\sigma} \frac{V_{x}-f_{w}}{V_{x x}-f_{w w}-2 f_{x w}} .
$$

We plug in $V=V_{0}+\epsilon V_{1}$ and $f=f_{0}+\epsilon f_{1}$,

$$
\begin{aligned}
\pi^{*} & =-\frac{\lambda}{\sigma} \frac{V_{0, x}+\epsilon h_{x} \gamma_{1}(x)}{V_{0, x x}+\epsilon h_{x x} \gamma_{1}(x)} \\
& =\frac{\lambda}{\sigma} R\left(1+\epsilon \frac{h_{x} \gamma_{1}(x)}{V_{0, x}}+o\left(\epsilon^{2}\right)\right)\left(1-\epsilon \frac{h_{x x} \gamma_{1}(x)}{V_{0, x x}}+o\left(\epsilon^{2}\right)\right) \\
& =\frac{\lambda}{\sigma} R\left[1+\epsilon \gamma_{1}(x)\left(\frac{h_{x}}{V_{0, x}}-\frac{h_{x x}}{V_{0, x x}}\right)\right]+o\left(\epsilon^{2}\right),
\end{aligned}
$$

where we denote $h:=\frac{\partial V_{0}}{\partial \gamma}$. Thus the equilibrium strategy will deviate from the optimal strategy in the case of constant risk aversion $\gamma_{0}$ by a fraction given by $\epsilon \gamma_{1}(x)\left(\frac{h_{x}}{V_{0, x}}-\frac{h_{x x}}{V_{0, x x}}\right)$.

\subsubsection{Power Utility Case}

Recall that the power utility function with constant risk aversion parameter $\gamma$ is:

$$
U(x)=\frac{x^{1-\gamma}}{1-\gamma}
$$

For the Merton problem with power utility and constant risk aversion, the value function $V(t, x)$ satisfies

$$
V_{t}-\frac{1}{2} \lambda^{2} \frac{V_{x}^{2}}{V_{x x}}=0
$$

with terminal condition $V(T, x)=\frac{x^{1-\gamma}}{1-\gamma}$. The solution for the PDE above is given by

$$
V(t, x)=\frac{x^{1-\gamma}}{1-\gamma} e^{\frac{\lambda^{2}}{2}\left(\frac{1-\gamma}{\gamma}\right)(T-t)} .
$$


This is our zeroth order value function $V_{0}(t, x)$ once we replace $\gamma$ with $\gamma_{0}$. We can find the first order correction by taking the partial derivative w.r.t. $\gamma$ and multiplying by $\gamma_{1}(x)$ :

$$
V_{1}(t, x)=\gamma_{1}(x)\left[\frac{1}{1-\gamma_{0}}-\log (x)-\frac{\lambda^{2}(T-t)}{2 \gamma_{0}^{2}}\right] V_{0}(t, x) .
$$

The equilibrium trading strategy up to first order is given by:

$$
\begin{aligned}
\pi^{*} & =\frac{\lambda}{\sigma \gamma_{0}}\left[1+\epsilon \gamma_{1}(x)\left(\frac{h_{x}}{V_{0, x}}-\frac{h_{x x}}{V_{0, x x}}\right)\right] \\
& =\frac{\lambda}{\sigma \gamma_{0}}\left[1+\epsilon \frac{\gamma_{1}(x)}{\gamma_{0}}\right] .
\end{aligned}
$$

We have provided some plots for the power utility case. Figure 1a compares a power utility function with constant risk aversion $\gamma=2$ to the one with risk aversion slowly decreasing with wealth. Here we have chosen $\gamma_{1}(x)=-\tan ^{-1}(x-10)$ and $\epsilon=0.01$ which retain the twice differentiability and concavity of the utility function. Figure $1 \mathrm{~b}$ compares the Merton optimal strategy with the equilibrium strategy up to the first order correction.

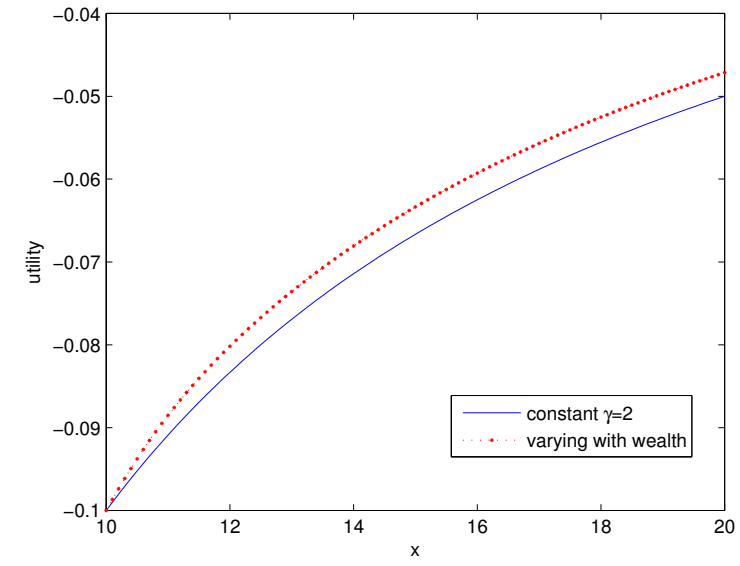

(a) Utility Functions

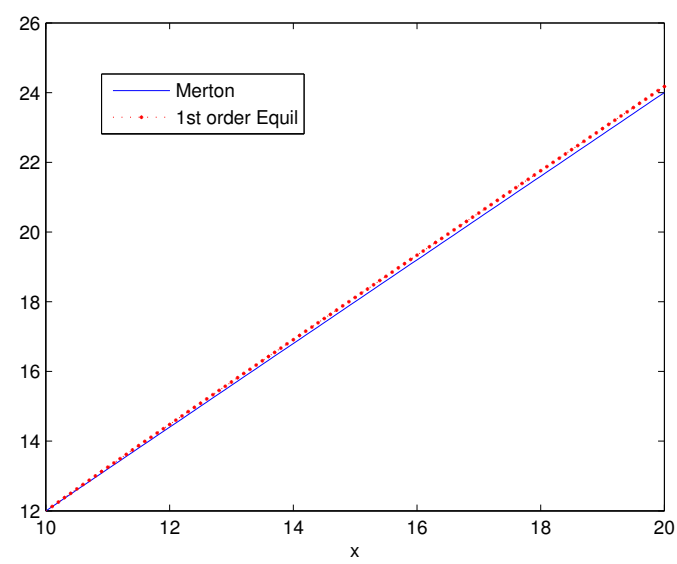

(b) Equilibrium Investment Strategies

Figure 1: Plots of utility functions and equilibrium trading strategies (up to 1st order correction) against wealth level in the case of power utility function. We chose $\mu=0.15, r=0, \sigma=0.25, \gamma_{0}=2, \gamma_{1}(x)=-\tan ^{-1}(x-10)$ and $\epsilon=0.01$. Risk aversion is modeled as slowly decreasing with wealth level here and the corresponding utility function is still concave and slightly above the utility function with constant risk aversion. Moreover, we see that the equilibrium strategy is slightly above the Merton strategy due to a lower risk aversion.

\subsection{Utility-indifference Pricing with Wealth-dependent Risk Aversion}

One of the immediate applications of wealth-dependent risk aversion is indifference pricing, where the (buyer's) price of the option is set such that the buyer has the same expected utility no matter he chooses to invest in a portfolio without the option or to invest in another portfolio with the option but paying a price at the beginning. In scenarios where the option is likely to cost a significant portion of the investor's wealth, for example constructing a power plant or start an $R \& D$ project as often considered in real option valuation 
problems, it is possible for the investor to become more risk averse after he purchases the option. A wealthdependent risk aversion can be used to capture this change. Here we look at the indifference pricing of an option written on a non-traded asset. The controlled wealth process follows

$$
d X_{t}^{\pi, x}=\pi_{t} d S_{t}^{(1)}+r\left(X_{t}^{\pi, x}-\pi_{t} S_{t}^{(1)}\right) d t
$$

where the price $S_{t}^{(1)}$ of the traded asset follows the geometric Brownian motion with drift $\mu$

$$
d S_{t}^{1}=\mu S_{t}^{(1)} d t+\sigma S_{t}^{(1)} d W_{t}^{(1)} .
$$

The option written on the non-traded asset $S_{t}^{(2)}$ has payoff $C\left(S_{T}^{(2)}\right)$ at terminal time $T$. And $S_{t}^{(2)}$ follows the SDE

$$
d S_{t}^{(2)}=p d t+q d W_{t}^{(2)}
$$

where $W_{t}^{(1)}$ and $W_{t}^{(2)}$ have correlation $\rho$. Now assuming $r=0$, the value function for the Merton problem without the option is

$$
\begin{aligned}
V(x, 0) & =-e^{-\gamma_{0} x-\lambda^{2} T / 2}+\epsilon \gamma_{1}(x) x e^{-\gamma_{0} x-\lambda^{2} T / 2}+o\left(\epsilon^{2}\right) \\
& =-e^{-\gamma_{0} x-\lambda^{2} T / 2}\left(1-\epsilon \gamma_{1}(x) x\right)+o\left(\epsilon^{2}\right) .
\end{aligned}
$$

Note that we are using exponential utility here to simplify the calculations. Now the value function with a long position in $k$ units of the option is given by

$$
\begin{aligned}
V\left(x-p_{k}, k\right)= & -e^{-\gamma_{0}\left(x-p_{k}\right)-\lambda^{2} T / 2}\left(1-\epsilon \gamma_{1}(x) x\right)\left(E^{Q_{0}}\left[e^{-k \gamma_{0}\left(1-\rho^{2}\right) C\left(S_{T}^{(2)}\right)}\left(1-k \epsilon \gamma_{1}(x)\left(1-\rho^{2}\right) C\left(S_{T}^{(2)}\right)\right)\right]\right)^{1 /\left(1-\rho^{2}\right)} \\
= & -e^{-\gamma_{0}\left(x-p_{k}\right)-\lambda^{2} T / 2}\left(1-\epsilon \gamma_{1}(x) x\right)\left(E^{Q_{0}}\left[e^{-k \gamma_{0}\left(1-\rho^{2}\right) C\left(S_{T}^{(2)}\right)}\right]\right)^{1 /\left(1-\rho^{2}\right)} \\
& +\epsilon k \gamma_{1}(x) e^{-\gamma_{0}\left(x-p_{k}\right)-\lambda^{2} T / 2}\left(E^{Q_{0}}\left[e^{-k \gamma_{0}\left(1-\rho^{2}\right) C\left(S_{T}^{(2)}\right)}\right]\right)^{\rho^{2} /\left(1-\rho^{2}\right)} E^{Q_{0}}\left[C\left(S_{T}^{(2)}\right) e^{-k \gamma_{0}\left(1-\rho^{2}\right) C\left(S_{T}^{(2)}\right)}\right],
\end{aligned}
$$

where $Q_{0}$ is the probability measure under which $S_{t}^{(2)}$ has a new drift $p-\rho \lambda q$ but the same diffusion $q$.

For the solution of $p_{k}$, we seek the following expansion:

$$
p_{k}=p_{k}^{(0)}+\epsilon p_{k}^{(\epsilon)}+o\left(\epsilon^{2}\right) .
$$

Consequently,

$$
\begin{aligned}
& V\left(x-p_{k}^{(0)}-\epsilon p_{k}^{(\epsilon)}, k\right)= \\
& V^{0}(x, 0) e^{\gamma_{0} p_{k}^{(0)}} \Theta_{T}^{\frac{1}{1-\rho^{2}}}\left[1+\epsilon\left(1-\gamma_{1}\left(x-p_{k}^{(0)}\right) x-\frac{k \gamma_{1}\left(x-p_{k}^{(0)}\right) E^{Q_{0}}\left[C\left(S_{T}^{(2)}\right) \Lambda_{T}\right]}{\Theta_{T}}\right)\right]+o\left(\epsilon^{2}\right)
\end{aligned}
$$

where we have used the following notation

$$
\begin{aligned}
V^{0}(x, 0) & :=-e^{-\gamma_{0} x-\lambda^{2} T / 2}, \\
\Lambda_{T} & :=e^{-k \gamma_{0}\left(1-\rho^{2}\right) C\left(S_{T}^{(2)}\right)}, \\
\Theta_{T} & :=E^{Q_{0}}\left[\Lambda_{T}\right],
\end{aligned}
$$


and that $\gamma_{1}\left(x-p_{k}^{(0)}-\epsilon p_{k}^{(\epsilon)}\right) \approx \gamma_{1}\left(x-p_{k}^{(0)}\right)$.

Now we just need to equate (18) and (20). At the zeroth order,

$$
-e^{-\gamma_{0} x-\lambda^{2} T / 2}=-e^{-\gamma_{0}\left(x-p_{k}^{(0)}\right)-\lambda^{2} T / 2} \Theta_{T}^{1 /\left(1-\rho^{2}\right)},
$$

from which we can find the zeroth order indifference price:

$$
p_{k}^{(0)}=-\frac{1}{\left(1-\rho^{2}\right) \gamma_{0}} \log \Theta_{T}
$$

At order $\epsilon$, after substituting in $p_{k}^{(0)}$, we have

$$
-\gamma_{1}(x) x=p_{k}^{(\epsilon)}-\gamma_{1}\left(x-p_{k}^{(0)}\right) x-k \gamma_{1}\left(x-p_{k}^{(0)}\right) \Theta_{T}^{-1} E^{Q_{0}}\left[C\left(S_{T}^{(2)}\right) \Lambda_{T}\right],
$$

from which we can get the order $\epsilon$ correction to the indifference price

$$
p_{k}^{(\epsilon)}=\left(\gamma_{1}\left(x-p_{k}^{(0)}\right)-\gamma_{1}(x)\right) x+\frac{k \gamma_{1}\left(x-p_{k}^{(0)}\right) E^{Q_{0}}\left[C\left(S_{T}^{(2)}\right) \Lambda_{T}\right]}{\Theta_{T}} .
$$

As we have assumed that the investor will become more risk averse when holding the option, i.e. $\gamma_{1}(x-$ $\left.p_{k}^{(0)}\right) \geq \gamma_{1}(x)$, the correction $p_{k}^{(\epsilon)}$ above tells us that the true indifference price will be higher than the constant risk aversion case for $k>0$ and $C(\cdot) \geq 0$.

\subsection{Stochastic Volatility Models with Volatility-dependent Risk Aversion}

Since first introduced by Hull and White [19] for pricing options, stochastic volatility models have gained wide popularity as they could reproduce features about the implied volatility surface that are missing in the standard Black-Scholes models. Incorporating the stochastic volatility framework is also one of the many extensions being studied recently for the classical Merton portfolio optimization problems.

One way to look at the problem is to use the timescale stochastic volatility asymptotics, which have been applied to many option pricing problems, see Fouque et al. [14] and the references therein. In this framework, the volatility is assumed to have a fast mean-reverting factor following a speeded-up diffusion process and/or a slow factor following a slowed-down diffusion. The empirical evidence to support the multiscale stochastic volatility model can be found in Chernov [8]. When Fouque et al. [15] treated the Merton problem in this way, they obtained in explicit forms both the fast and slow scale corrections to the value function, which resemble the stochastic volatility corrections for pricing European-style options.

A natural question to ask is whether volatility, an indicator of investment risk, would affect the risk aversion parameter, a measure of investor's attitude towards risk. This question is nontrivial when the assumption of constant volatility has been dropped. Intuitively the answer should be yes, as investors would usually focus on preserving capitals when the risky assets have high volatility, thus becoming more risk averse. Empirical results also support this argument, which can be found in Scheicher [27] and Tarashev et al. [30]. Scheicher [27] discovered a positive relationship between the implied risk aversion in German equity market and the implied volatility of the US market, and Tarashev et al. [30] concluded from results obtained in different equity and fixed income markets that a higher risk aversion is linked to higher volatilities and this is more noticeable in the equity markets.

In the next section, we will look at the case where risk aversion is a function of the slow scale volatility factor so it slowly varies. Our rationale is that the effect from the fast scale factor would likely be averaged 
out over the investment horizon if it is long enough; and it is the general trend of the volatility that would reflect the change in investors' risk aversion. We will derive the extended HJB system and carry out the slow scale asymptotic expansion in this case to approximate the value function and equilibrium strategy. Using power utility functions, we will show that it is possible to obtain results similar to Fouque et al. [15] but with an additional correction term to account for change in risk aversion.

\subsubsection{Slow Scale Stochastic Volatility Model}

Suppose that the volatility slowly fluctuates as a general diffusion process and the stock price follows a geometric Brownian Motion as usual:

$$
\left\{\begin{array}{l}
d S_{t}=\mu S_{t} d t+\sigma\left(Z_{t}\right) S_{t} d W_{t} \\
d Z_{t}=\delta c\left(Z_{t}\right) d t+\sqrt{\delta} g\left(Z_{t}\right) d W_{t}^{\prime}
\end{array}\right.
$$

where $W_{t}$ and $W_{t}^{\prime}$ are Brownian motions with correlation $\rho^{\prime} \in(-1,1)$. We have the wealth process:

$$
d X_{t}=\pi_{t} \mu d t+\pi_{t} \sigma\left(Z_{t}\right) d W_{t}
$$

and the associated infinitesimal generator:

$$
\mathcal{A}^{\pi_{t}}(t, x, z)=\partial_{t}+\pi_{t} \mu(z) \partial_{x}+\frac{1}{2} \pi_{t}^{2} \sigma(z)^{2} \partial_{x}^{2}+\delta c(z) \partial_{z}+\frac{1}{2} \delta g(z)^{2} \partial_{z}^{2}+\sqrt{\delta} \pi_{t} \rho g(z) \sigma(z) \partial_{x z}^{2} .
$$

The portfolio optimization problem we consider here is:

$$
V(t, x, z)=\sup _{\boldsymbol{\pi}} \mathbb{E}_{t, x, z}\left[U\left(X_{T}^{\boldsymbol{\pi}}, \gamma\left(Z_{t}\right)\right)\right],
$$

where we make the risk aversion dependent on current level of the slow factor $Z_{t}$. The extended HJB system for the value function can be derived in the same way as the wealth-dependent risk aversion case (with a two dimensional state process now), which is given by

$$
\left\{\begin{array}{r}
\sup _{\pi_{t}}\left\{V_{t}+\pi_{t} \mu(z) V_{x}+\frac{1}{2} \pi_{t}^{2} \sigma(z)^{2} V_{x x}+\sqrt{\delta} \rho \pi_{t} g(z) \sigma(z)\left(V_{x z}-f_{x w}\right)\right. \\
\left.+\delta c(z)\left(V_{z}-f_{w}\right)+\frac{1}{2} \delta g(z)^{2}\left(V_{z z}-f_{w w}-2 f_{w z}\right)\right\}=0 \\
f_{t}+\pi_{t}^{*} \mu(z) f_{x}+\frac{1}{2} \pi_{t}^{* 2} \sigma(z)^{2} f_{x x}+\delta c(z) f_{z}+\frac{1}{2} \delta g(z)^{2} f_{z z}+\sqrt{\delta} \rho \pi_{t}^{*} g(z) \sigma(z) f_{x z}=0
\end{array}\right.
$$

with terminal conditions:

$$
\left\{\begin{aligned}
V(T, x, z) & =U(x, \gamma(z)) \\
f(T, x, z, w) & =U(x, \gamma(w)) .
\end{aligned}\right.
$$

By the first order conditions, the equilibrium strategy takes the form:

$$
\pi_{t}^{*}=-\left.\frac{\mu(z) V_{x}+\sqrt{\delta} \rho g(z) \sigma(z)\left[V_{x z}-f_{x w}\right]}{\sigma(z)^{2} V_{x x}}\right|_{w=z} .
$$


Plugging this equilibrium strategy back to the extended HJB system, we get:

$$
\left\{\begin{array}{l}
V_{t}-\frac{\left\{\mu(z) V_{x}+\sqrt{\delta} \rho g(z) \sigma(z)\left[V_{x z}-f_{x w}\right]\right\}^{2}}{2 \sigma(z)^{2} V_{x x}}+\delta\left\{c(z)\left(V_{z}-f_{w}\right)+\frac{g(z)^{2}}{2}\left[V_{z z}-f_{w w}-2 f_{z w}\right]\right\}=0 \\
f_{t}-\frac{\mu(z) V_{x}+\sqrt{\delta} \rho g(z) \sigma(z)\left[V_{x z}-f_{x w}\right]}{\sigma(z)^{2} V_{x x}}\left[\mu(z) f_{x}+\sqrt{\delta} \rho g(z) \sigma(z) f_{x z}\right] \\
\quad+\frac{\left\{\mu(z) V_{x}+\sqrt{\delta} \rho g(z) \sigma(z)\left[V_{x z}-f_{x w}\right]\right\}^{2}}{2 \sigma(z)^{2} V_{x x}^{2}} f_{x x}+\delta\left[c(z) f_{z}+\frac{g(z)^{2}}{2} f_{z z}\right]=0 .
\end{array}\right.
$$

Now we assume that the risk aversion $\gamma\left(Z_{t}\right)$ takes the form:

$$
\gamma\left(Z_{t}\right)=\gamma_{0}+\sqrt{\delta} \gamma_{1}\left(Z_{t}\right)+o(\delta)
$$

thus slowly varies with the slow scale volatility factor. And we expand $V$ and $f$ as:

$$
\begin{aligned}
V(t, x, z) & =V_{0}(t, x, z)+\sqrt{\delta} V_{1}(t, x, z)+\delta V_{2}(t, x, z)+o\left(\delta^{\frac{3}{2}}\right) \\
f(t, x, z, w) & =f_{0}(t, x, z, w)+\sqrt{\delta} f_{1}(t, x, z, w)+\delta f_{2}(t, x, z, w)+o\left(\delta^{\frac{3}{2}}\right) .
\end{aligned}
$$

Now introduce the risk tolerance function:

$$
R:=R(t, x, z)=-\frac{V_{0, x}(t, x, z)}{V_{0, x x}(t, x, z)},
$$

and the differential operator:

$$
\mathcal{D}_{k}:=R^{k} \frac{\partial^{k}}{\partial x^{k}}
$$

as well as the linear operator:

$$
\mathcal{L}_{t, x, z}=\partial_{t}+\lambda(z)^{2} \mathcal{D}_{1}+\frac{1}{2} \lambda(z)^{2} \mathcal{D}_{2}
$$

where $\lambda(z):=\frac{\mu}{\sigma(z)}$ denotes the Sharpe ratio.

We can find that $V_{0}(t, x, z)=f_{0}(t, x, z, z)$ is the Merton value function with Sharpe ratio fixed at $\lambda(z)$ and risk aversion parameter fixed at $\gamma_{0}$. As a result, order $\sqrt{\delta}$ equations become:

$$
\left\{\begin{aligned}
\mathcal{L}_{t, x, z} V_{1}+\rho g(z) \lambda(z) \mathcal{D}_{1}\left(V_{0, z}-f_{0}, w\right. & =0 \\
\mathcal{L}_{t, x, z} f_{1}+\rho g(z) \lambda(z) \mathcal{D}_{1} f_{0, z} & =0
\end{aligned}\right.
$$

with terminal condition given by:

$$
\left\{\begin{aligned}
V_{1}(T, x, z) & =\gamma_{1}(z) \frac{\partial U}{\partial \gamma}\left(x, \gamma_{0}\right) \\
f_{1}(T, x, z, w) & =\gamma_{1}(w) \frac{\partial U}{\partial \gamma}\left(x, \gamma_{0}\right) .
\end{aligned}\right.
$$

Proposition 3. The solution to (30) is given by:

$$
V_{1}(t, x, z)=\gamma_{1}(z) V_{0, \gamma}+\frac{1}{2}(T-t) \rho \lambda(z) g(z) \mathcal{D}_{1} V_{0, z}
$$


Proof. Using the result from Lemma 1, we can see that $\gamma_{1}(w) f_{0, \gamma}$ is a solution to the PDE problem below:

$$
\left\{\begin{aligned}
\mathcal{L}_{t, x, z} f_{1} & =0 \\
f_{1}(T, x, z, w) & =\gamma_{1}(w) \frac{\partial U}{\partial \gamma}\left(x, \gamma_{0}\right)
\end{aligned}\right.
$$

which is the original order $\sqrt{\delta}$ PDE problem without the source term. Now if we can find the solution to the full PDE problme with zero terminal condition, we can find the full solution satisfying the original terminal condition by combining the two partial solutions. This can be done by making use of the "Vega-Gamma" relation in Lemma 3.1 of Fouque et al. [15], which states

$$
f_{0, z}=-(T-t) \lambda(z) \lambda^{\prime}(z) \mathcal{D}_{2} f_{0}
$$

The second problem can be rewritten as follows

$$
\left\{\begin{aligned}
\mathcal{L}_{t, x, z} f_{1} & =(T-t) \rho g(z) \lambda(z)^{2} \lambda^{\prime}(z) \mathcal{D}_{1} \mathcal{D}_{2} f_{0} \\
f_{1}(T, x, z, w) & =0
\end{aligned}\right.
$$

Using the commutativity property of $\mathcal{L}_{t, x, z}$ with $\mathcal{D}_{1}$ and the equality $\mathcal{D}_{1} f_{0}=-\mathcal{D}_{2} f_{0}$ from [15], we find that

$$
\begin{aligned}
f_{1}(t, x, z, w) & =-\frac{1}{2}(T-t)^{2} \rho g(z) \lambda(z)^{2} \lambda^{\prime}(z) \mathcal{D}_{1} \mathcal{D}_{2} f_{0} \\
& =\frac{1}{2}(T-t) \rho \lambda(z) g(z) \mathcal{D}_{1} f_{0, z}
\end{aligned}
$$

is the solution. We get the solution for $V(t, x, z)$ by combining the two partial solutions and replacing the $w$ variable with $z$.

Once we get the solution of $V_{1}$, the equilibrium trading strategy up to order $o(\sqrt{\delta})$ can be computed:

$$
\pi^{*}=\frac{\lambda(z)}{\sigma(z)} R-\sqrt{\delta}\left\{\frac{\rho g(z)}{\sigma(z)} \frac{V_{0, x z}}{V_{0, x x}}+\frac{\lambda(z)}{\sigma(z)}\left[\frac{V_{1, x}}{V_{0, x x}}+R \frac{V_{1, x x}}{V_{0, x x}}\right]\right\} .
$$

Power Utility Case For a power utility function:

$$
U(x, \gamma(z))=\frac{1}{1-\gamma(z)} x^{1-\gamma(z)}
$$

we have the zeroth order value function given by:

$$
V_{0}(t, x, z)=\frac{x^{1-\gamma_{0}}}{1-\gamma_{0}} e^{\frac{\lambda(z)^{2}}{2} \frac{1-\gamma_{0}}{\gamma_{0}}(T-t)} .
$$

Thus the explicit form of the first order value function is:

$$
V_{1}(t, x, z)=\left\{\frac{(T-t)^{2} \rho g(z) \lambda(z)^{2} \lambda^{\prime}(z)\left(1-\gamma_{0}\right)^{2}}{2 \gamma_{0}^{2}}+\gamma_{1}(z)\left[\frac{1}{1-\gamma_{0}}-\log (x)-\frac{\lambda(z)^{2}(T-t)}{2 \gamma_{0}^{2}}\right]\right\} V_{0}(t, x, z)
$$


The strategy is given by:

$$
\pi^{*}=\frac{\lambda(z) x}{\sigma(z) \gamma_{0}}+\sqrt{\delta} \underbrace{\left[\frac{\rho g(z) \lambda^{\prime}(z) \lambda(z)\left(1-\gamma_{0}\right)(T-t)}{\sigma(z) \gamma_{0}^{2}}\right.}_{\text {slow factor adjustment in [15] }}-\underbrace{\left.\frac{\lambda(z)}{\sigma(z)} \frac{\gamma_{1}(z)}{\gamma_{0}}\left(1+\frac{1}{\gamma_{0}}\right)\right]}_{\text {risk aversion adjustment }} x .
$$

We now compare the Merton optimal strategy, the optimal strategy with first order correction for the slow volatility factor appeared in [15] and our equilibrium strategy with first order correction. Note that the second strategy is equivalent to (36) with only the first fractional term inside the square bracket. We notice that for different levels of the slow factor, the proportions that the two adjustment factors would contribute to the first order correction are different. Figure 2 contains the plots of the three strategies for different ranges of the slow factor. Figures $2 \mathrm{a}$ and $2 \mathrm{c}$ show that for small $z$, the main contributor of the first order correction is the first fractional term inside the square bracket of (71), whereas for larger values of $z$, as Figures $2 b$ and $2 \mathrm{~d}$ suggest, an increasing risk aversion plays the major role instead. The direction to which the first adjustment factor affects the strategy depends on the sign of the correlation factor $\rho$.

\subsection{Comparison with Mixture of Power Utility Functions}

A mixture of power utility functions takes the following form:

$$
U^{m i x}(x)=c_{1} \frac{x^{1-\gamma_{1}}}{1-\gamma_{1}}+c_{2} \frac{x^{1-\gamma_{2}}}{1-\gamma_{2}},
$$

as introduced in Fouque et al. [15], where $\gamma_{1} \neq \gamma_{2}$ and $c_{1}, c_{2}$ are positive constants. Under this utility function, the relative risk aversion is not constant any more but decreases in $x$. Now let us look at a power utility function with wealth-dependent risk aversion:

$$
U(x)=\frac{x^{1-\gamma(x)}}{1-\gamma(x)}
$$

We can choose $\gamma(x)$ to make $U^{\text {mix }}(x)=U(x)$, but in the case of power utility the solution will be a complex-valued function due to the presence of $\gamma(x)$ in the exponent of $x$. (In contrast, for a mixture of exponential utility functions, $\gamma(x)$ will be real-valued).

For the case of small wealth-dependence, we have the following expansion:

$$
\begin{aligned}
U(x) & =\frac{x^{1-\left(\gamma_{0}+\epsilon \gamma_{1}+o\left(\epsilon^{2}\right)\right)}}{1-\left(\gamma_{0}+\epsilon \gamma_{1}+o\left(\epsilon^{2}\right)\right)} \\
& =\frac{x^{1-\gamma_{0}}-\epsilon \gamma_{1} \log (x) x^{1-\gamma_{0}}+o\left(\epsilon^{2}\right)}{1-\gamma_{0}}\left(1+\epsilon \frac{\gamma_{1}}{1-\gamma_{0}}+o\left(\epsilon^{2}\right)\right) \\
& =\frac{x^{1-\gamma_{0}}}{1-\gamma_{0}}+\epsilon\left\{-\frac{x^{1-\gamma_{0}}}{1-\gamma_{0}} \gamma_{1} \log (x)+\frac{x^{1-\gamma_{0}}}{1-\gamma_{0}} \frac{\gamma_{1}}{1-\gamma_{0}}\right\}++o\left(\epsilon^{2}\right)
\end{aligned}
$$

where $\gamma_{1} \equiv \gamma_{1}(x)$ can be chosen in such a way that the expansion is also a mixture of power utility functions. For example, we can set $\gamma_{1}(x)$ to be:

$$
\gamma_{1}(x)=\frac{c_{1} x^{k_{1}}+c_{2} x^{k_{2}}}{-\log (x)+\frac{1}{1-\gamma_{0}}}
$$




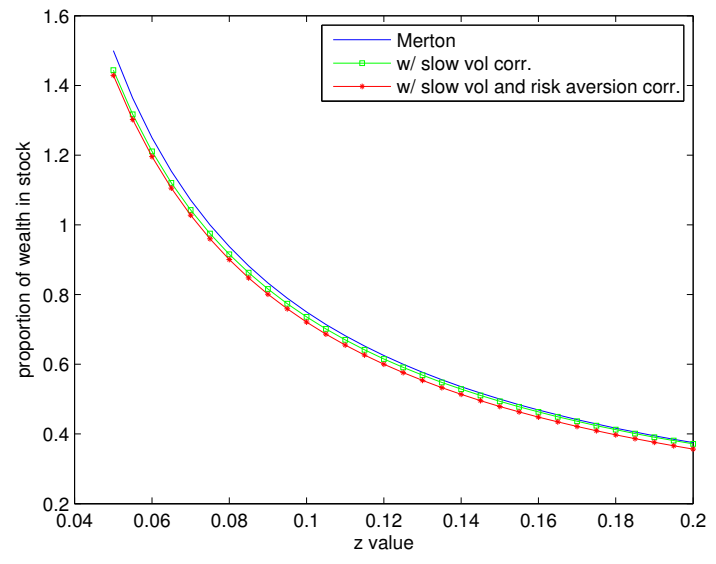

(a) $\rho=-0.2$

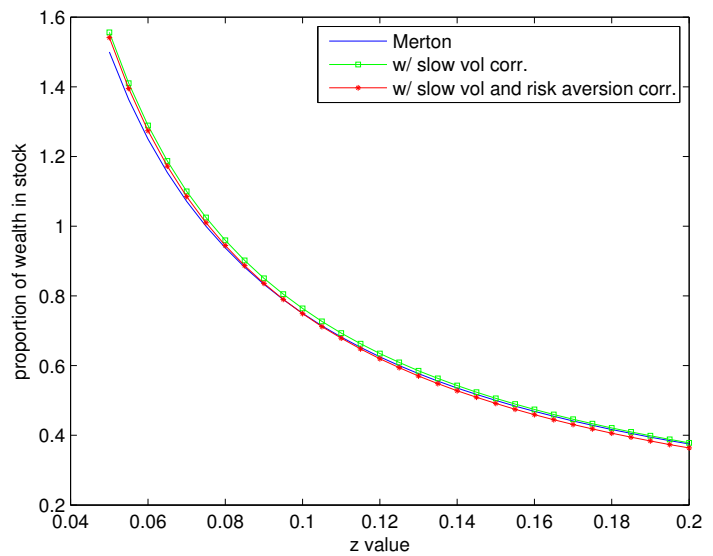

(c) $\rho=0.2$

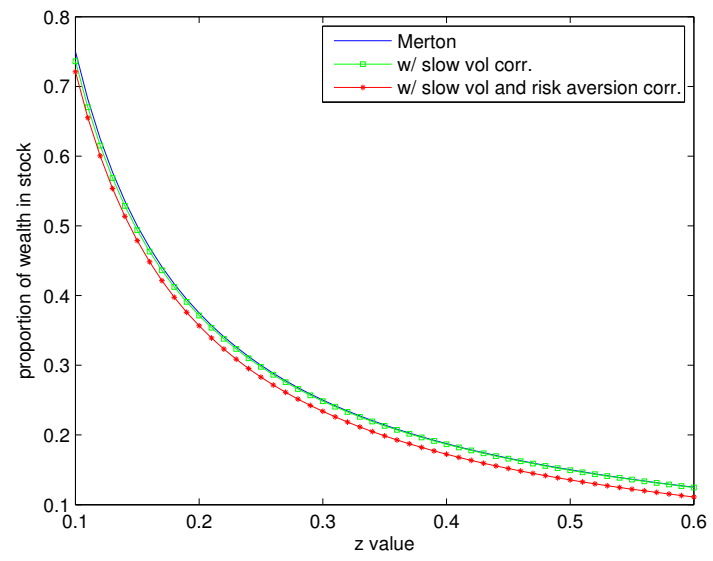

(b) $\rho=-0.2$

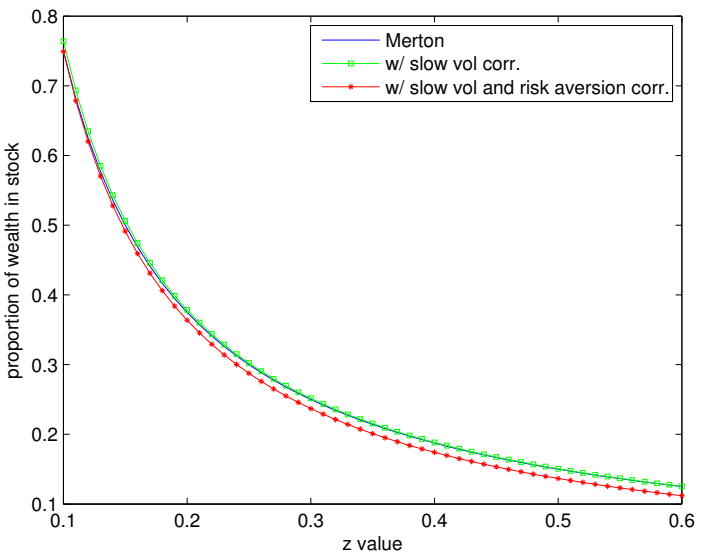

(d) $\rho=0.2$

Figure 2: Plots of the equilibrium strategies, in terms of the proportion of total wealth, against the slow stochastic volatility parameter $z$, in the case of power utility function. We chose the stochastic volatility model to be slow scale $\operatorname{CIR}\left(\right.$ Heston), with $\mu=0.15, r=0, \sigma(z)=\sqrt{z}, g(z)=\frac{\sqrt{z}}{2}, \rho= \pm 0.2, T=5, \gamma_{0}=2, \gamma_{1}=\tan ^{-1}(z)$ and the time scale is $\delta=0.1$.

then for $x$ belonging to the region where $\gamma_{1}(x)$ is bounded, the expansion above becomes:

$$
\begin{aligned}
& \frac{x^{1-\gamma_{0}}}{1-\gamma_{0}}+\epsilon \frac{x^{1-\gamma_{0}}}{1-\gamma_{0}}\left(c_{1} x^{k_{1}}+c_{2} x^{k_{2}}\right)+o\left(\epsilon^{2}\right) \\
= & \frac{x^{1-\gamma_{0}}}{1-\gamma_{0}}+\epsilon c_{1} \frac{x^{1-\gamma_{0}+k_{1}}}{1-\gamma_{0}}+\epsilon c_{2} \frac{x^{1-\gamma_{0}+k_{2}}}{1-\gamma_{0}}+o\left(\epsilon^{2}\right) \\
= & \frac{x^{1-\gamma_{0}}}{1-\gamma_{0}}+\epsilon c_{1} \frac{1-\gamma_{0}+k_{1}}{1-\gamma_{0}} \frac{x^{1-\gamma_{0}+k_{1}}}{1-\gamma_{0}+k_{1}}+\epsilon c_{2} \frac{1-\gamma_{0}+k_{2}}{1-\gamma_{0}} \frac{x^{1-\gamma_{0}+k_{2}}}{1-\gamma_{0}+k_{2}}+o\left(\epsilon^{2}\right)
\end{aligned}
$$

i.e. a mixture of three power utility functions up to order $\epsilon$.

Despite the similarity between the two types of utility functions, i.e. the terminal conditions for the two problems, the portfolio optimization under a mixture of power utility functions remains time-consistent as 
the risk aversion always depends on the terminal wealth, which is a random variable revealed at time $T$. In our problem here, we have made $\gamma(\cdot)$ dependent on the instantaneous level of wealth which becomes the source of time inconsistency.

\section{Investment/Consumption Problems with Non-exponential Discounting}

In the previous section we have looked at the utility maximization for terminal wealth with time-varying risk aversions by using the method of asymptotic expansions. Here we want to study the investment/consumption problem under non-exponential discounting. We adopt the same two-asset diffusion model (1) for this problem thus we have our wealth process being

$$
d X_{t}=\left[\pi_{t}(\mu-r) X_{t}+\left(r X_{t}-c_{t}\right)\right] d t+\pi_{t} \sigma X_{t} d W_{t},
$$

where the additional term $c_{t}$ denotes our instantaneous consumption rate and $\pi_{t}$ is the proportion of wealth invested in the risky asset. We define the objective function as:

$$
J(t, x, \pi, \mathbf{c})=\mathbb{E}_{t, x}\left[\int_{t}^{T} \varphi(s-t) U\left(c_{s}\right) d s+\varphi(T-t) U\left(X_{T}^{\pi, \mathbf{c}}\right)\right],
$$

where $U(\cdot)$ is some appropriate utility function to be chosen and $\varphi(\cdot)$ is the discount function for the utility derived from consumption. We do not require $\varphi(\cdot)$ to be exponential, which is the source of time inconsistency for this problem. As usual, the value function is defined as:

$$
V(t, x)=\sup _{\pi, \mathbf{c}} J(t, x, \pi, \mathbf{c})
$$

Similar to the utility maximization for terminal wealth case, we have the following result as a consequence of Definition 2.4:

Proposition 4. The value function $V(t, x)$ satisfies the following HJB-type equation:

$$
\begin{aligned}
\sup _{\pi, c \in \mathbb{R} \times \mathbb{R}_{+}}\left\{\frac{\partial V}{\partial t}+[\pi x(\mu-r)+(r x\right. & \left.-c)] \frac{\partial V}{\partial x}+\frac{\pi^{2}}{2} \sigma^{2} x^{2} \frac{\partial^{2} V}{\partial x^{2}}+U(c)\right\}= \\
& -\mathbb{E}_{t, x}\left[\int_{t}^{T} \varphi^{\prime}(s-t) U\left(c_{s}^{*}\right) d s+\varphi^{\prime}(T-t) U\left(X_{T}^{\pi, \mathbf{c}^{*}}\right)\right]
\end{aligned}
$$

where we have terminal and boundary conditions given by

$$
\begin{aligned}
V(T, x) & =0 \\
V(t, 0) & =0,
\end{aligned}
$$

and $c_{s}^{*}$ denotes the equilibrium consumption in the future time $s \geq t$.

Proof. For this proof we ignore the $\varphi(T-t) U\left(X_{T}^{\pi, \mathbf{c}^{*}}\right)$ term for simplicity. Using the definition of equilibrium strategies in (4), let us define:

$$
\pi_{s}^{\epsilon}=\left\{\begin{array}{l}
\pi \text { for } s \in[t, t+\epsilon] \\
\pi^{*} \text { for } s \in(t+\epsilon, T]
\end{array} \text { and } \mathbf{c}_{s}^{\epsilon}=\left\{\begin{array}{l}
c \text { for } s \in[t, t+\epsilon] \\
\mathbf{c}^{*} \text { for } s \in(t+\epsilon, T] .
\end{array}\right.\right.
$$


i.e. our policy $\mathbf{u}:=\left(\pi_{s}^{\epsilon}, c_{s}^{\epsilon}\right)_{s \in[t, T]}$ is defined such that it is a uniform and arbitrary perturbation from $\mathbf{u}^{*}$ for the period $[t, t+\epsilon]$ and the two strategy will coincide after $t+\epsilon$. Therefore we have

$$
J\left(t+\epsilon, X_{t+\epsilon}, \mathbf{u}\right)=V\left(t+\epsilon, X_{t+\epsilon}\right),
$$

which we take the expectation conditional on $(t, x)$ and plug into the following inequality:

$$
\begin{aligned}
V(t, x) & \geq J(t, x, \mathbf{u}) \\
& =J(t, x, \mathbf{u})-\mathbb{E}_{t, x}\left[J\left(t+\epsilon, X_{t+\epsilon}, \mathbf{u}\right)\right]+\mathbb{E}_{t, x}\left[V\left(t+\epsilon, X_{t+\epsilon}\right)\right] \\
& =\mathbb{E}_{t, x}\left[\int_{t}^{T} \varphi(s-t) U\left(c_{s}^{\epsilon}\right) d s-\int_{t+\epsilon}^{T} \varphi(s-t-\epsilon) U\left(c_{s}^{*}\right) d s\right]+\mathbb{E}_{t, x}\left[V\left(t+\epsilon, X_{t+\epsilon}\right)\right] \\
& \approx \epsilon \mathbb{E}_{t, x}\left[U\left(c_{t+\epsilon}^{\epsilon}\right)-\int_{t+\epsilon}^{T} \varphi^{\prime}(s-t-\epsilon) U\left(c_{s}^{\epsilon}\right) d s\right]+\mathbb{E}_{t, x}\left[V\left(t+\epsilon, X_{t+\epsilon}\right)\right]
\end{aligned}
$$

which in turn is a result of the following simple Taylor expansion for point $t$ around $(t+\epsilon)$ :

$$
\left.\int_{t}^{T} \varphi(s-t) U\left(c_{s}^{\epsilon}\right) d s \approx \int_{t+\epsilon}^{T} \varphi(s-t-\epsilon) U\left(c_{s}^{\epsilon}\right) d s+(-\epsilon)\left(-U\left(c_{t+\epsilon}^{\epsilon}\right)-\int_{t+\epsilon}^{T} \varphi^{\prime}(s-t-\epsilon) U\left(c_{s}^{\epsilon}\right) d s\right)\right)+o\left(\epsilon^{2}\right) .
$$

Dividing the inequality by $\epsilon$ and taking the limit $\epsilon \rightarrow 0$, we obtain:

$$
\mathcal{G}^{\pi, c} V(t, x)+U\left(c_{t}\right)+\mathbb{E}_{t, x}\left[\int_{t}^{T} \varphi^{\prime}(s-t) U\left(c_{s}^{*}\right) d s\right] \leq 0
$$

where $\mathcal{G}^{\pi, c}$ denotes the infinitesimal generator for $V(t, x)$. If we take the supremum over $\pi$ and $c$, the inequality above becomes equality and we recover the HJB-type equation for $V(t, x)$ less the $\mathbb{E}\left[\varphi^{\prime}(T-\right.$ t) $\left.U\left(X_{T}^{\pi, \mathbf{c}^{*}}\right)\right]$ term, which can be obtained using the same argument as above. The boundary conditions are straightforward.

Remark 3.1. A first look may suggest that the result (41) above contradicts the remark made in Section 2.1.3 regarding the two-equation characteristics for time inconsistency, since this time we only have one HJB-type equation. In fact, the two-equation feature is masked in the term $\mathbb{E}_{t, x}\left[\int_{t}^{T} \varphi^{\prime}(s-t) U\left(c_{s}^{*}\right) d s\right]$, which characterizes the difference on how one's current self and his immediate future self would value consumption. This is equivalent to saying that the derivative characterizes the difference between the current value function and the continuation value function. If we take the discounting function to be of exponential type, then the term $\mathbb{E}_{t, x}\left[\int_{t}^{T} \varphi^{\prime}(s-t) U\left(c_{s}^{*}\right) d s\right]$ will simply reduce to $-r V(t, x)$, where $r$ is the exponential discounting rate; and the HJB-type equation will reduce to the classical HJB equation for an investment/consumption problem. However, for all non-exponential-type discounting functions, $\mathbb{E}_{t, x}\left[\int_{t}^{T} \varphi^{\prime}(s-t) U\left(c_{s}^{*}\right) d s\right]$ makes the equation non-local and thus hard to solve. See Ekeland et al. [13] for a numerical treatment of a similar problem using backward integration.

\subsection{Approximating a Hyperbolic Discount Function}

On one hand, the exponential discounting produces explicit solutions but is less realistic. On the other hand, a hyperbolic discount function is in accordance with how people behave but becomes less tractable. There is a clear trade-off between tractability and realisticity. Consider the following discount function:

$$
\varphi^{\alpha}(\tau)=e^{(\alpha-1) \delta_{0} \tau-\alpha \log \left(1+\delta_{1} \tau\right)}
$$


for $\alpha \in[0,1]$. When $\alpha=0$, this is an exponential discount function with discount rate $\delta_{0}$. When $\alpha=1$, this is a hyperbolic discount function with rate $\delta_{1}$. For $\alpha \in(0,1)$, the discount function will have partial amount of the features that a hyperbolic discount function has.

Now we consider the case where $\alpha=\epsilon>0$ is very small, then

$$
\varphi^{\epsilon}(\tau) \approx e^{-\delta_{0} \tau}\left(1+\epsilon \Delta(\tau)+o\left(\epsilon^{2}\right)\right),
$$

where $\Delta(\tau)=\delta_{0} \tau-\log \left(1+\delta_{1} \tau\right)$ (we can choose other forms of $\Delta(\tau)$ as well). This discount function will allow us to solve the HJB-type equation (41) using asymptotic expansions in the following subsection. Figure 3 illustrates that this discount function is close to the exponential discounting case for small $\epsilon$ while it bends towards the hyperbolic discount function. Thus it mimics the hyperbolic discounting feature by a small amount controlled by $\epsilon$.

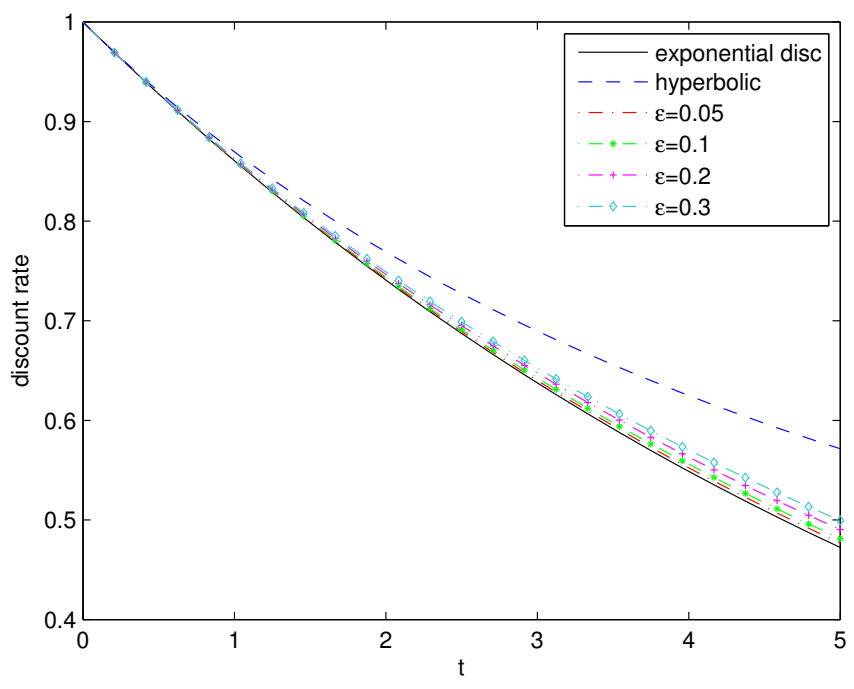

Figure 3: A comparison of discount functions $\exp \left(-\delta_{0} \tau\right), 1 /\left(1+\delta_{1} \tau\right)$ and the one defined by (43) with $\delta_{0}=\delta_{1}=$ 0.15 for various values of $\epsilon$.

\subsection{Solving the HJB-type Equation Using Asymptotic Expansions}

Let us go back to the HJB type equation (41). Using the first order conditions, the maximizations over $\pi$ and $c$ can be done separately:

$$
\pi^{*}=-\frac{\mu-r}{x \sigma^{2}} \frac{V_{x}}{V_{x x}} \text { and } c^{*}=\left(U^{\prime}\right)^{-1}\left(V_{x}\right)
$$

where $V_{x}$ denotes the first derivative w.r.t $x$ and so on. We can see that $c^{*}$ is the Legendre transform of the utility function at $V_{x}$. From now on we will adopt a power utility function with risk aversion $\gamma$ :

$$
U(c)=\frac{c^{1-\gamma}}{1-\gamma}
$$


thus we have $c^{*}=\left(V_{x}\right)^{-1 / \gamma}$. We plug $\pi^{*}$ and $c^{*}$ into (41) to obtain the following nonlinear non-local PDE:

$$
V_{t}-\frac{\lambda^{2}}{2} \frac{V_{x}^{2}}{V_{x x}}+\frac{\gamma}{1-\gamma}\left(V_{x}\right)^{\frac{\gamma-1}{\gamma}}+r x V_{x}=-\mathbb{E}_{t, x}\left[\int_{t}^{T} \varphi^{\prime}(s-t) U\left(c_{s}^{*}\right) d s+\varphi^{\prime}(T-t) U\left(X_{T}^{\pi, \mathbf{c}^{*}}\right)\right],
$$

with boundary conditions $V(T, x)=0$ and $V(t, 0)=0$.

As a consequence of the expansion (43) for the discount function, we seek a similar expansion for the value function:

$$
V(t, x)=V_{0}(t, x)+\epsilon V_{1}(t, x)+o\left(\epsilon^{2}\right),
$$

which we plug into (45). After grouping terms of different orders, we have the following PDEs for the first two orders:

$$
\begin{array}{r}
V_{0, t}-\frac{\lambda^{2}}{2} \frac{V_{0, x}^{2}}{V_{0, x x}}+\frac{\gamma}{1-\gamma}\left(V_{0, x}\right)^{\frac{\gamma-1}{\gamma}}+r x V_{0, x}-\delta_{0} V_{0}=0, \\
V_{1, t}-\left(\lambda^{2} \frac{V_{0, x}}{V_{0, x x}}+\left(V_{0, x}\right)^{-\frac{1}{\gamma}}-r x\right) V_{1, x}+\frac{\lambda^{2}}{2} \frac{V_{0, x}^{2}}{V_{0, x x}^{2}} V_{1, x x}-\delta_{0} V_{1}= \\
-\mathbb{E}_{t, x}\left[\int_{t}^{T} \Delta^{\prime}(s-t) e^{-\delta_{0}(s-t)} \frac{\left[c_{0, s}^{*}\left(X_{s}^{(0)}\right)\right]^{1-\gamma}}{1-\gamma} d s+\Delta^{\prime}(T-t) \frac{\left(X_{T}^{(0)}\right)^{1-\gamma}}{1-\gamma}\right],
\end{array}
$$

where $X_{s}^{(0)}$ denotes the wealth process under the zeroth order equilibrium investment and consumption strategies $\pi_{0}^{*}$ and $c_{0}^{*}$. The detail of the decomposition of (45) into (47) can be found in the Appendix.

Note The first equation in (47) can be solved in a fairly standard way with the appropriate boundary conditions. Once this is solved, we obtain the zeroth order value function as well as the zeroth order strategies that will give explicit forms for the parameters of the second equation. As we will see later, the solution to the second PDE can be found explicitly. We have therefore managed to bypass the "nonlocal" issue in the HJB-type PDE by using asymptotic expansions. This allows us to avoid the usual numerical procedures as seen for example, in [13].

\subsubsection{Zeroth Order Solution}

The solution to the zeroth order equation with zero terminal \& boundary conditions is very well-known. Using separation of variables method, we seek solution $V_{0}(t, x)$ of the following form:

$$
V_{0}(t, x)=\frac{x^{1-\gamma}}{1-\gamma}[f(t)]^{\gamma} .
$$

The original PDE problem reduces to the following ODE problem

$$
f^{\prime}(t)+\frac{1-\gamma}{\gamma}\left(\frac{\lambda^{2}}{2 \gamma}+r\right) f(t)+e^{\frac{\delta_{0}}{\gamma} t}=0
$$

with $f(T)=1$. Thus we have

$$
f(t)=\frac{-e^{A_{2} t}+A_{3} e^{A_{2} T+A_{1}(T-t)}}{A_{1}+A_{2}}
$$


where $A_{1}=\frac{1-\gamma}{\gamma}\left(\frac{\lambda^{2}}{2 \gamma}+r\right), A_{2}=\frac{\delta_{0}}{\gamma}$ and $A_{3}=\frac{A_{1}+A_{2}+e^{A_{2} T}}{e^{A_{2} T}}$. We can also compute the zeroth order equilibrium strategies:

$$
\pi_{0}^{*}=\frac{\lambda}{\sigma \gamma} \text { and } c_{0}^{*}=\frac{x}{f(t)} .
$$

\subsubsection{First Order Solution}

Using the preceding result, we can simplify the first order PDE from (47) into:

$$
\begin{aligned}
V_{1, t}+\left(\frac{\lambda^{2}}{\gamma}+r-1\right) x V_{1, x} & +\frac{\lambda^{2}}{2 \gamma^{2}} x^{2} V_{1, x x}-\delta_{0} V_{1}= \\
\mathbb{E}_{t, x} & {\left[\int_{t}^{T} \Delta^{\prime}(s-t) e^{-\delta_{0}(s-t)} \frac{\left[c_{0, s}^{*}\left(X_{s}^{(0)}\right)\right]^{1-\gamma}}{1-\gamma} d s+\Delta^{\prime}(T-t) \frac{\left(X_{T}^{(0)}\right)^{1-\gamma}}{1-\gamma}\right] . }
\end{aligned}
$$

In order to deal with the expectation term on the right side, we need the dynamics of the zeroth order wealth process $X_{t}^{(0)}$ under zeroth order equilibrium strategies:

$$
d X_{t}^{(0)}=\left(\pi_{0}^{*}(\mu-r)+r-f(t)^{-1}\right) X_{t}^{(0)} d t+\pi_{0}^{*} \sigma X_{t}^{(0)} d W_{t}
$$

which we notice is a lognormal process and we can write out the expectation term explicitly.

It follows that

$$
\mathbb{E}_{0, x}\left[\frac{\left(X_{t}^{(0)}\right)^{1-\gamma}}{1-\gamma}\right]=\frac{x^{1-\gamma}}{1-\gamma} e^{(1-\gamma)\left[\pi_{0}^{*}(\mu-r)+r-f(t)^{-1}-\frac{\gamma}{2} \pi_{0}^{* 2} \sigma^{2}\right] t}
$$

Therefore, (52) becomes

$$
V_{1, t}+\left(\frac{\lambda^{2}}{\gamma}+r-1\right) x V_{1, x}+\frac{\lambda^{2}}{2 \gamma^{2}} x^{2} V_{1, x x}=\delta_{0} V_{1}+\frac{x^{1-\gamma}}{1-\gamma} F(t)
$$

where $F(t)$ denotes the integral:

$$
F(t):=\int_{t}^{T} \Delta^{\prime}(s-t) e^{-\delta_{0}(s-t)} e^{(1-\gamma)\left[\pi_{0}^{*}(\mu-r)+r-f(s)^{-1}-\frac{\gamma}{2} \pi_{0}^{* 2} \sigma^{2}\right] s} d s .
$$

The ansatz $V_{1}(t, x)=\frac{x^{1-\gamma}}{1-\gamma} g(t)$ reduces $(55)$ to a first order ODE problem:

$$
g^{\prime}(t)+\left[\left(\frac{\lambda^{2}}{2 \gamma}+r-1\right)(1-\gamma)-\delta_{0}\right] g(t)=F(t)
$$

with terminal condition $g(T)=0$, which has a solution given by:

$$
g(t)=\int_{t}^{T} F(s) e^{B_{1}(s-t)} d s,
$$

where $B_{1}:=\left(\frac{\lambda^{2}}{2 \gamma}+r-1\right)(1-\gamma)-\delta_{0}$. 


\subsubsection{First Order Corrections for Equilibrium Strategies}

Proposition 5. We have the following respective first order corrections (to multiply by $\epsilon$ ) to the equilibrium strategies:

$$
\pi_{1}^{*}=0 \text { and } c_{1}^{*}=-\frac{1}{\gamma} \frac{g(t)}{f(t)} c_{0}^{*}
$$

Proof. We have

$$
V_{0}(t, x)=U(x) f(t) \text { and } V_{1}(t, x)=U(x) g(t)
$$

where $U(x)$ is the power utility function with risk aversion $\gamma$. For the equilibrium proportion of wealth invested in the risky asset, we have

$$
\pi^{*} \approx-\frac{\mu-r}{x \sigma^{2}} \frac{V_{0, x}+\epsilon V_{1, x}}{V_{0, x x}+\epsilon V_{1, x x}}=-\frac{\mu-r}{x \sigma^{2}} \frac{U^{\prime}(x)}{U^{\prime \prime}(x)} \frac{(f(t)+\epsilon g(t))}{(f(t)+\epsilon g(t))}=\frac{\mu-r}{\gamma \sigma^{2}} \equiv \pi_{0}^{*},
$$

whereas for the equilibrium consumption rate, we have

$$
c^{*} \approx\left(V_{0, x}+\epsilon V_{1, x}\right)^{-\frac{1}{\gamma}}=\left(V_{0, x}\right)^{-\frac{1}{\gamma}}\left[1-\frac{\epsilon}{\gamma} \frac{V_{1, x}}{V_{0, x}}+o\left(\epsilon^{2}\right)\right]=c_{0}^{*}\left(1-\frac{\epsilon}{\gamma} \frac{g(t)}{f(t)}\right) .
$$

We have found that adding a small amount of hyperbolic-discounting feature to the discount function does not change the proportion of wealth invested in the risky asset, while it will affect the consumption rate by a fraction depending on the ratio $\frac{g(t)}{f(t)}$. Figure 4 illustrates how the approximated equilibrium strategies change over time compared to the optimal one in the exponential discounting case. In general, we find that hyperbolic discounting would encourage one to consume at a faster rate. The fact that $g(t)$ is negative also means that the value function is more negative compared to the exponential discounting case, indicating a loss of welfare. For relatively larger values of $\epsilon$, the equilibrium strategy is clearly non-monotonic. More precisely, the ideal consumption speed starts at some higher level compared to the exponential discounting case and it has a decreasing trend at the beginning. But eventually the consumption speed will start to increase monotonically once we are sufficiently far away from the commencing point $t=0$. In fact, this non-monotonicity feature agrees with the consumption pattern observed in real-life household data, which is one of the main reasons economists support the use of hyperbolic discounting. We also note that similar results were obtained in [13] in which the authors made use of backward numerical integration techniques to solve the full extended HJB equation analogous to (41).

\subsection{A Bound for the Value Function: Infinite Horizon Case}

In this section we want to illustrate some characteristics of the hyperbolic discounting problem using Laplace transform. Suppose we have an infinite horizon investment/consumption problem instead:

$$
V(x)=\sup _{\mathbf{c}} \mathbb{E}\left[\int_{0}^{\infty} \frac{1}{1+\delta t} \frac{c_{t}^{1-\gamma}}{1-\gamma} d t\right] .
$$

The following equation holds for the hyperbolic discount function by Laplace transform:

$$
\frac{1}{1+\delta t}=\int_{0}^{\infty} \frac{e^{-\tau\left(t+\frac{1}{\delta}\right)}}{\delta} d \tau .
$$




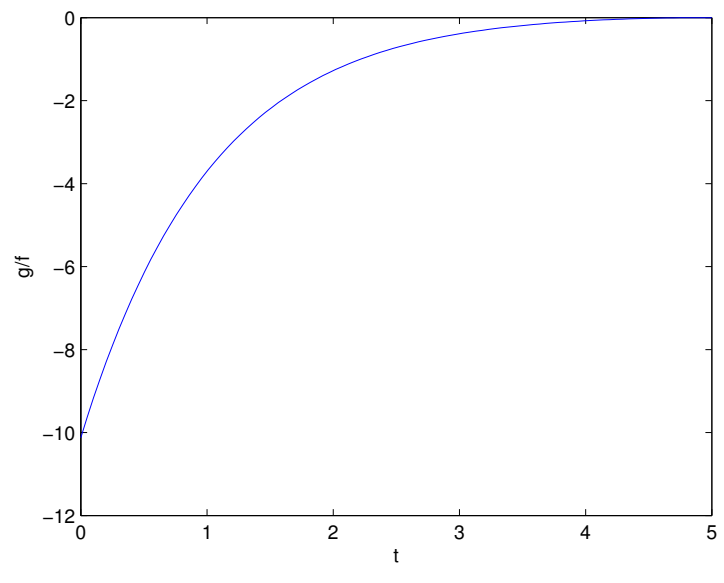

(a) The ratio $\frac{g(t)}{f(t)}$.

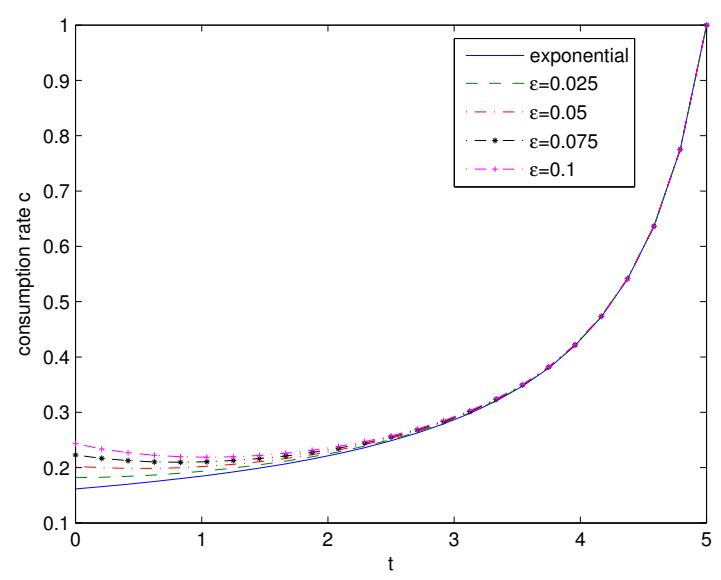

(b) Consumption rates.

Figure 4: $\mu=0.15, r=0.05, \sigma=0.25, \gamma=2, \delta_{0}=\delta_{1}=0.15$ and $T=5$. We have included the utility of wealth at time $T$ here to fix the unbounded consumption rate near $T$.

Therefore we have

$$
\begin{aligned}
V(x) & =\sup _{\mathbf{c}} \int_{0}^{\infty} \mathbb{E}\left[\int_{0}^{\infty} e^{-\tau t} \frac{c_{t}^{1-\gamma}}{1-\gamma} d t\right] \frac{e^{-\frac{\tau}{\delta}}}{\delta} d \tau \\
& =\sup _{\mathbf{c}} \int_{0}^{\infty} \bar{J}(x, c, \tau) \frac{e^{-\frac{\tau}{\delta}}}{\delta} d \tau \\
& \leq \int_{0}^{\infty} \sup _{\mathbf{c}} \bar{J}(x, \mathbf{c}, \tau) \frac{e^{-\frac{\tau}{\delta}}}{\delta} d \tau \\
& =\beta C(x) \int_{0}^{\infty} \frac{e^{-\beta \tau}}{(\tau+\alpha)^{\gamma}} d \tau,
\end{aligned}
$$

where $C(x):=\frac{\gamma^{\gamma} x^{1-\gamma}}{1-\gamma}, \alpha:=-\delta(1-\gamma)-\frac{\lambda^{2}(1-\gamma)}{2 \gamma}, \beta=\frac{1}{\delta}$ and $\bar{J}(x, c, \tau)$ denotes the objective function for the infinite horizon investment problem under consumption $c$ and exponential discount rate $\tau$, in which case the value function has an explicit solution.

The second line of (60) best illustrates how time inconsistency arises from hyperbolic discounting. Loosely speaking, the integral can be seen as the weighted average of a continuum of optimization problems parameterized by the (exponential-type) discount rate $\tau$. If there is a policy $\mathbf{c}^{*}$ that can maximize all the objective functions, then the inequality becomes an equality and we can say $\mathbf{c}^{*}$ is the optimal-for-all policy. Unfortunately, the optimal-for-all policy does not exist most of the time. Nevertheless we can still find a policy $\mathbf{c}^{* *}$ that maximizes the integral, i.e. a linearization of the objectives. And it turns out this particular policy $\mathbf{c}^{* *}$ is a Pareto optimum that corresponds to a point on the Pareto front of the multi-objective optimization problem. Consequently, the difference between two sides of the inequality corresponds to the distance between a strict optimal value and the Pareto-optimal value under the particular linearization given.

The integral in the last line of (60) can be solved for positive-integer-valued $\gamma=n \in \mathbb{Z}_{+}$

$$
\int_{0}^{\infty} \frac{e^{-\beta \tau}}{(\tau+\alpha)^{\gamma}} d \tau=-n ! \sum_{j=0}^{n} \frac{\alpha^{j}}{j !} .
$$


Thus we have produced a bound for the value function in case $\gamma$ is a positive integer

$$
V(x) \leq-\beta n ! \sum_{j=0}^{n} \frac{\alpha^{j}}{j !} C(x) .
$$

\subsection{Extension with Proportional Transaction Costs}

We extend our study to the situation where proportional transaction cost exists. The dynamics of the portfolio can be represented as below:

$$
\begin{aligned}
& d X_{t}^{(b)}=\left(r X_{t}^{(b)}-\hat{c}_{t}\right) d t-(1+\kappa) d \hat{L}_{t}+(1-\lambda) d \hat{M}_{t} \\
& d X_{t}^{(s)}=\mu X_{t}^{(s)} d t+\sigma X_{t}^{(s)} d W_{t}+d \hat{L}_{t}-d \hat{M}_{t},
\end{aligned}
$$

where $X_{t}^{(b)}$ and $X_{t}^{(s)}$ represent the wealth in the risk-free bank account and in the risky asset (stock) respectively. Again $\hat{c}_{t}$ is the rate of consumption and $d \hat{L}_{t}:=\hat{l}_{t} d t$ and $d \hat{M}_{t}:=\hat{m}_{t} d t$ denote the purchase and sell of the risky asset which will incur proportional transaction costs $\kappa$ and $\lambda$ respectively.

Our objective function has now been modified into maximizing consumption utility over an infinite horizon because we want to make the analysis simpler. The objective function is given by

$$
J\left(x^{(b)}, x^{(s)}, \hat{c}, \hat{l}, \hat{m}\right)=\mathbb{E}\left[\int_{0}^{\infty} \varphi(s) U\left(c_{s}\right) d s \mid X_{0}^{(b)}=x^{(b)}, X_{0}^{(s)}=x^{(s)}\right],
$$

given the current level of wealth $x^{(b)}$ in the bank account and $x^{(s)}$ in the stock as well as the admissible controls $\hat{c}, \hat{m}, \hat{l}$, where the utility function $U($.$) is still chosen to be the power type. Now define the value$ function:

$$
V\left(x^{(b)}, x^{(s)}\right)=\sup _{\hat{c}, \hat{m}, \hat{l}} J\left(x^{(b)}, x^{(s)}, \hat{c}, \hat{l}, \hat{m}\right) .
$$

Almost identical to the result from Proposition 4, the value function satisfies the HJB-type equation:

$$
\begin{aligned}
\sup _{\hat{c}, \hat{m}, \hat{l}}\left(r x^{(b)}-\hat{c}\right) V_{x^{(b)}}+\mu x^{(s)} V_{x^{(1)}} & +\frac{1}{2} \sigma^{2}\left(x^{(s)}\right)^{2} V_{x^{(s)} x^{(s)}}+\left[(1-\lambda) V_{x^{(b)}}-V_{\left.x^{(s)}\right)}\right] \hat{m} \\
& +\left[V_{x^{(1)}}-(1+\kappa) V_{x^{(b)}}\right] \hat{l}+U(\hat{c})=-\mathbb{E}_{x^{(b)} x^{(s)}}\left[\int_{0}^{\infty} \varphi^{\prime}(s) U\left(\hat{c}_{s}^{*}\right) d s\right],
\end{aligned}
$$

only this time there is no time derivative. When $\varphi(\cdot)$ is exponential type, this becomes the HJB equation that was probably first derived by Davis and Norman [10], who noticed that the desirable strategies for purchase and sell were "bang-bang" type which only took place on the boundaries of the no-transaction region at maximum possible rates.

The homothetic property holds for the value function since we have chosen a power utility function, meaning that

$$
V\left(\rho x^{(b)}, \rho x^{(s)}\right)=\rho^{1-\gamma} V\left(x^{(b)}, x^{(s)}\right),
$$

for any positive constant $\rho$. Thus we can write the value function $V\left(x^{(b)}, x^{(s)}\right)$ into

$$
V\left(x^{(b)}, x^{(s)}\right)=\left(x^{(s)}\right)^{1-\gamma} V\left(x^{(b)} / x^{(s)}, 1\right):=\left(x^{(s)}\right)^{1-\gamma} \Phi\left(x^{(b)} / x^{(s)}\right) .
$$


As a consequence, it is sufficient to study the transformed value function $\Phi(z)$ where we use $z$ to denote the ratio $x^{(b)} / x^{(s)}$.

The problem reduces to a free boundary ODE problem:

$$
\begin{aligned}
& \left(\mu-\frac{1}{2} \sigma^{2} \gamma\right)(1-\gamma) \Phi(z)+\left(r-\mu+\sigma^{2} \gamma\right) z \Phi^{\prime}(z)+\frac{1}{2} \sigma^{2} z^{2} \Phi^{\prime \prime}(z) \\
& +\frac{\gamma}{1-\gamma}\left[\Phi^{\prime}(z)\right]^{-(1-\gamma) / \gamma}+\mathbb{E}_{z}\left[\int_{0}^{\infty} \varphi^{\prime}(s) \frac{\left[\Phi^{\prime}\left(Z_{s}\right)\right]^{-(1-\gamma) / \gamma}}{1-\gamma} d s\right]=0
\end{aligned}
$$

with free boundary conditions:

$$
\begin{array}{r}
\Phi^{\prime}(l)(1-\lambda+l)-(1-\gamma) \Phi(l)=0 \\
\Phi^{\prime}(u)(1+\kappa+u)-(1-\gamma) \Phi(u)=0,
\end{array}
$$

where the upper and lower boundaries $u$ and $l$ are to be determined. The ODE (68) is difficult to solve because it involves a free boundary as well as a non-local term $\mathbb{E}_{z}\left[\int_{0}^{\infty} \varphi^{\prime}(s) \frac{\left[\Phi^{\prime}\left(Z_{s}\right)\right]^{-(1-\gamma) / \gamma}}{1-\gamma} d s\right]$ that is the source of time inconsistency. Again let us deal with it using the asymptotic approximation method. We assume the same expansion for the discount function $\varphi(\cdot)$ as in (43). And we seek an expansion for the solution $\Phi(z)$ of the following form:

$$
\Phi(z)=\Phi_{0}(z)+\epsilon \Phi_{1}(z)+o\left(\epsilon^{2}\right) .
$$

At zeroth order, we need to solve the free boundary ODE:

$$
\left\{\begin{aligned}
\beta_{0} \Phi_{0}+\beta_{1} z \Phi_{0}^{\prime}+\beta_{2} z^{2} \Phi_{0}^{\prime \prime}+\frac{\gamma}{1-\gamma}\left[\Phi_{0}^{\prime}\right]^{\frac{\gamma-1}{\gamma}} & =0 \\
\Phi_{0}^{\prime}\left(l_{0}\right)\left(1-\lambda+l_{0}\right)-(1-\gamma) \Phi_{0}\left(l_{0}\right) & =0 \\
\Phi_{0}^{\prime}\left(u_{0}\right)\left(1+\kappa+u_{0}\right)-(1-\gamma) \Phi_{0}\left(u_{0}\right) & =0
\end{aligned}\right.
$$

with $l_{0}, u_{0}$ to be determined, where $\beta_{0}, \beta_{1}$ and $\beta_{2}$ are constant parameters defined as

$$
\beta_{0}:=\left(\mu-\frac{1}{2} \sigma^{2} \gamma\right)(1-\gamma)-\delta_{0}, \quad \beta_{1}:=r-\mu+\sigma^{2} \gamma, \quad \beta_{2}:=\frac{1}{2} \sigma^{2} .
$$

At first order, we need to solve a fixed boundary ODE problem, but with a nonlocal term:

$$
\left\{\begin{aligned}
\beta_{0} \Phi_{1}+\left[\beta_{1} z+\frac{\gamma}{1-\gamma}\left(\Phi_{0}^{\prime}\right)^{-\frac{1}{\gamma}}\right] \Phi_{1}^{\prime}+\beta_{2} z^{2} \Phi_{1}^{\prime \prime}+\mathbb{E}_{z}\left[\int_{0}^{\infty} e^{-\delta_{0} s} \Delta^{\prime}(s) \frac{\left(\Phi_{0}^{\prime}\right)^{\frac{\gamma-1}{\gamma}}}{1-\gamma} d s\right] & =0 \\
\Phi_{1}^{\prime}\left(l_{0}\right)\left(1-\lambda+l_{0}\right)-(1-\gamma) \Phi_{1}\left(l_{0}\right) & =0 \\
\Phi_{1}^{\prime}\left(u_{0}\right)\left(1+\kappa+u_{0}\right)-(1-\gamma) \Phi_{1}\left(u_{0}\right) & =0
\end{aligned}\right.
$$

from which we can compute the first order corrections to the NT boundary as

$$
\begin{aligned}
l_{1} & =-\frac{\left(1-\lambda+l_{0}\right) \Phi_{1}^{\prime \prime}\left(l_{0}\right)+\gamma \Phi_{1}^{\prime}\left(l_{0}\right)}{\left(1-\lambda+l_{0}\right) \Phi_{0}^{\prime \prime \prime}\left(l_{0}\right)+(1+\gamma) \Phi_{0}^{\prime \prime}\left(l_{0}\right)} \\
u_{1} & =-\frac{\left(1+\kappa+u_{0}\right) \Phi_{1}^{\prime \prime}\left(u_{0}\right)+\gamma \Phi_{1}^{\prime}\left(u_{0}\right)}{\left(1+\kappa+u_{0}\right) \Phi_{0}^{\prime \prime \prime}\left(u_{0}\right)+(1+\gamma) \Phi_{0}^{\prime \prime}\left(u_{0}\right)},
\end{aligned}
$$

which are derived from the original boundary equations. 


\subsubsection{Zeroth Order Solution}

The zeroth order problem (71) is exactly the original problem in [10], which has been shown to have a solution that can be written as

$$
\Phi_{0}(z)=\frac{1}{1-\gamma}\left[\frac{1-\gamma}{\gamma} h_{1}(z)\right]^{-\gamma}\left(\frac{z}{h_{2}(z)}\right)^{1-\gamma},
$$

where $h_{2}(z)$ and $h_{1}(z)$ solve the system below

$$
\begin{aligned}
h_{2}^{\prime}(z) & =\frac{1}{\beta_{2} z}\left[R\left(h_{2}(z)\right)-h_{1}(z)\right] \\
h_{1}^{\prime}(z) & =\frac{1-\gamma}{\gamma} \frac{h_{1}(z)}{\beta_{2} z h_{2}(z)}\left[h_{1}(z)-Q\left(h_{2}(z)\right)\right],
\end{aligned}
$$

with boundary conditions

$h_{2}\left(l_{0}\right)=\frac{l_{0}}{l_{0}+1-\lambda}, \quad h_{1}\left(l_{0}\right)=Q\left(\frac{l_{0}}{l_{0}+1-\lambda}\right), \quad h_{2}\left(u_{0}\right)=\frac{u_{0}}{u_{0}+1+\kappa}, \quad h_{1}\left(u_{0}\right)=Q\left(\frac{u_{0}}{u_{0}+1+\kappa}\right)$,

where we define $Q(x):=-\frac{\beta_{0}}{1-\gamma}-\beta_{1} x+\beta_{2} \gamma x^{2}$ and $R(x):=Q(x)+\beta_{2}(1-x) x$. This ODE system (75) can be solved numerically using a shooting method as suggested by [10].

\subsubsection{First Order Solution}

Recall (72), in order to obtain $\Phi_{1}(z)$, we need to solve a fixed boundary ODE, which is numerically straightforward except for the source term

$$
\mathbb{E}_{z}\left[\int_{0}^{\infty} e^{-\delta_{0} s} \Delta^{\prime}(s) \frac{\left(\Phi_{0}^{\prime}\left(Z_{s}\right)\right)^{\frac{\gamma-1}{\gamma}}}{1-\gamma} d s\right],
$$

which involves a path integral depending on the process $Z_{t} \equiv \frac{X_{t}}{Y_{t}}$. Note that the major issue here is that we do not have an explicit form for $\Phi_{0}$ as it is computed numerically, whereas the nonlocality issue has disappeared similar to the case without transaction cost because of the expansion we have used. To approximate the source term we reply on Monte Carlo method to generate a large number of sample paths for $Z_{t}$ up to some time $T$ and evaluate the truncated integral for each of these paths using Riemann-sum approximation, after which the estimated expectation can be obtained by taking the average. We first use Ito's Lemma to get the dynamics for the process $Z_{s}$ under the zeroth order equilibrium strategies $c_{0}^{*}, d L_{0}^{*}$ and $d M_{0}^{*}$ :

$$
d Z_{t}=\left[\left(r-\mu+\frac{\sigma^{2}}{2}\right) Z_{t}-c_{0, t}^{*}\right] d t-\sigma Z_{t} d W_{t}-\left(1+\kappa+Z_{t}\right) d L_{0, t}^{*}+\left(1-\lambda+Z_{t}\right) d M_{0, t}^{*} .
$$

To further simplify the problem we put restrictions on the "Bang-Bang" type strategies $d L_{0}^{*}$ and $d M_{0}^{*}$ so that the process $Z_{t}$ diffuses within the zeroth order NT region but whenever it hits the boundary $l_{0}$ or $u_{0}$, it will be pushed back to the Merton ratio line in the NT region. Figure 5 gives a few sample path of the controlled process $X_{s}$. We repeat the approximations for a grid of initial values $z$ and we can smooth out the results using Fourier-type curve fitting method.

We are left with a second-order ODE with a mixed-type boundary condition to solve. Numerical discretization makes it a linear system of equations $A x=b$ with $A$ being a tridiagonal matrix. Once we solve this, we can compute the first-order corrections for the NT boundaries as well as for the equilibrium strategies. 


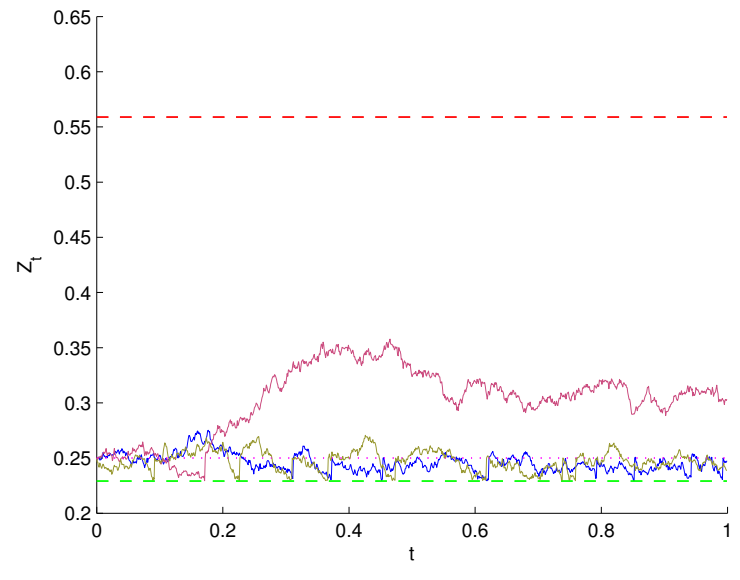

(a) $Z_{s}, \sigma=0.25$

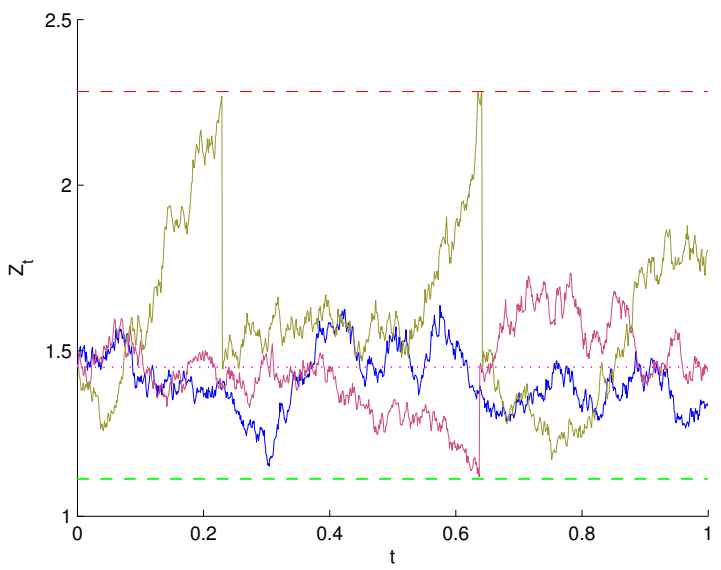

(b) $Z_{s}, \sigma=0.35$

Figure 5: Some realizations of $Z_{s}$ with zeroth order optimal consumption rate $c_{0}^{*}$ and boundaries $l_{0}$ and $u_{0}$. Note that each time the process hits the boundaries, it will be pushed back to the Merton line inside the NT region.

\subsubsection{Numerical Results}

We have numerically solved the zeroth and first order ODE problems using the following set of parameter values: $r=0.05, \mu=0.15, \gamma=2, \kappa=\lambda=0.01, \delta_{0}=\delta_{1}=0.15$ or 0.3 and $\sigma=0.25: 0.02$ : 0.35. Figure 6 gives illustrations for the zeroth order value function $\Phi_{0}(z)$ and the zeroth order equilibrium consumption rate $c_{0}^{*}(z)$. For different volatility $\sigma$, the NT boundaries are different. Figure 7 illustrates the NT region with/without first order corrections. We can see that hyperbolic discounting has the effect of shrinking the NT region, which leads to more frequent trading and rebalancing. This result matches the behavior of typical individual investors who tend to be myopic and impatient and are therefore prone to excessive rebalancing of their investment portfolios. However, whether this is a good or bad thing requires further investigation on this problem.

\section{Conclusion}

In this article, we have studied several time-inconsistent problems related to portfolio optimization. By using asymptotic methods, we can handle the nonlocality issue that arises from the game-theoretic methodology framework introduced to tackle time-inconsistency. Tractable solutions have been obtained in situations where the time-inconsistent problems can be closely approximated by time-consistent ones, which can also provide a qualitative/directional characterization of the equilibrium investment strategies in more general cases. Our results are intuitive and can describe how differently investors behave in reality and in time consistent settings. 


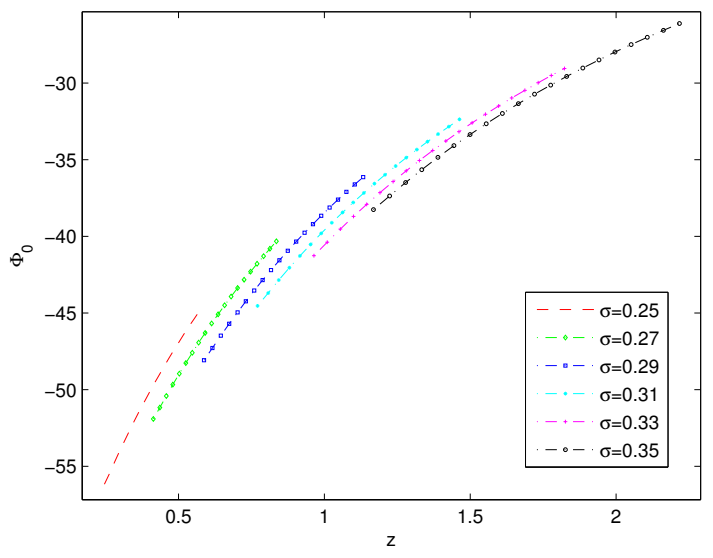

(a) $\Phi_{0}(z), \delta_{0}=\delta_{1}=0.15$

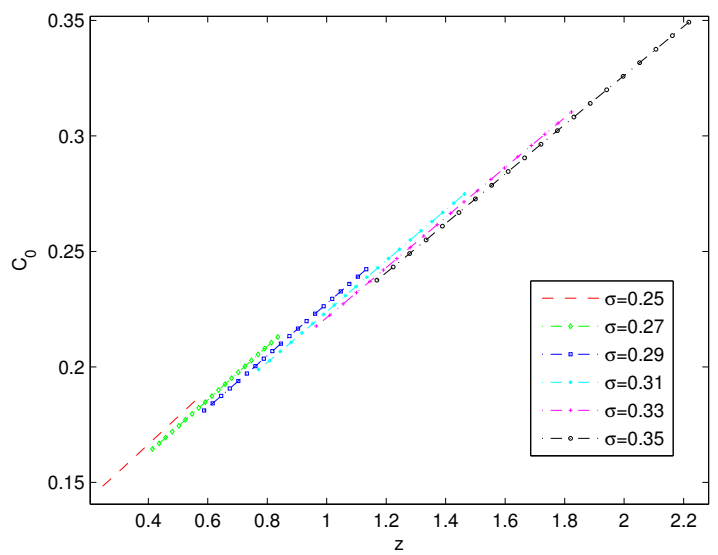

(c) $c_{0}^{*}, \delta_{0}=\delta_{1}=0.15$

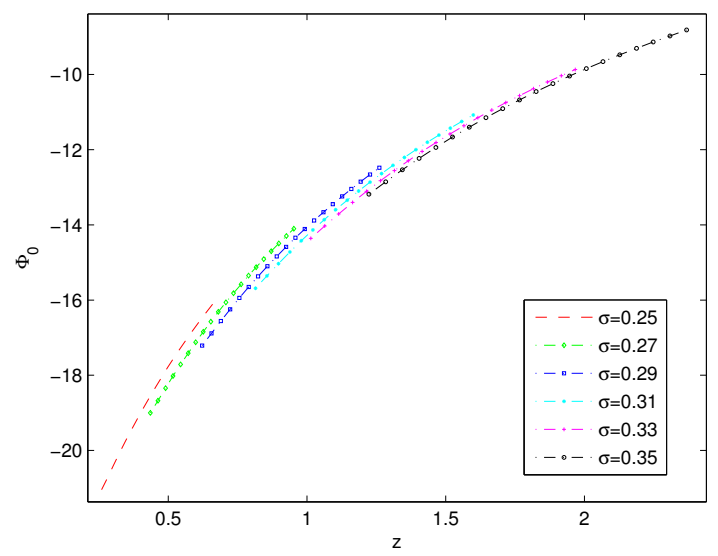

(b) $\Phi_{0}(z), \delta_{0}=\delta_{1}=0.3$

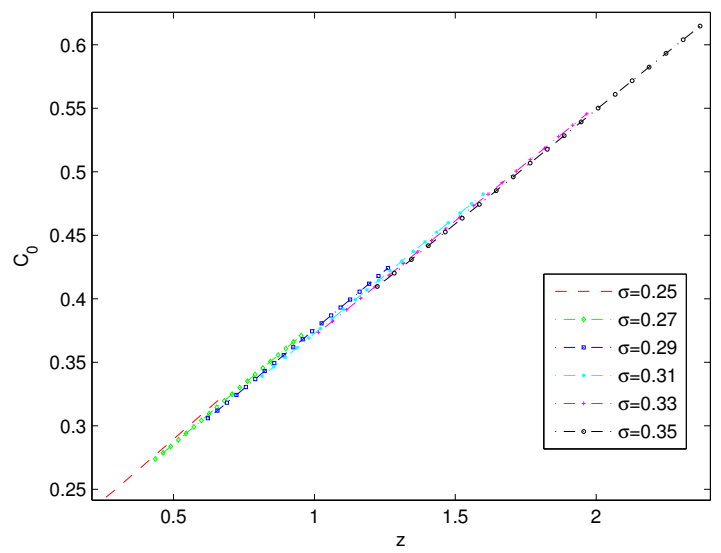

(d) $c_{0}^{*}, \delta_{0}=\delta_{1}=0.3$

Figure 6: Plots of the zeroth value function $\Phi_{0}(z)$ and optimal consumption rate $c_{0}^{*}(z)$ for parameter values $r=0.05, \mu=0.15, \gamma=2$ and $\kappa=\lambda=0.01$. 


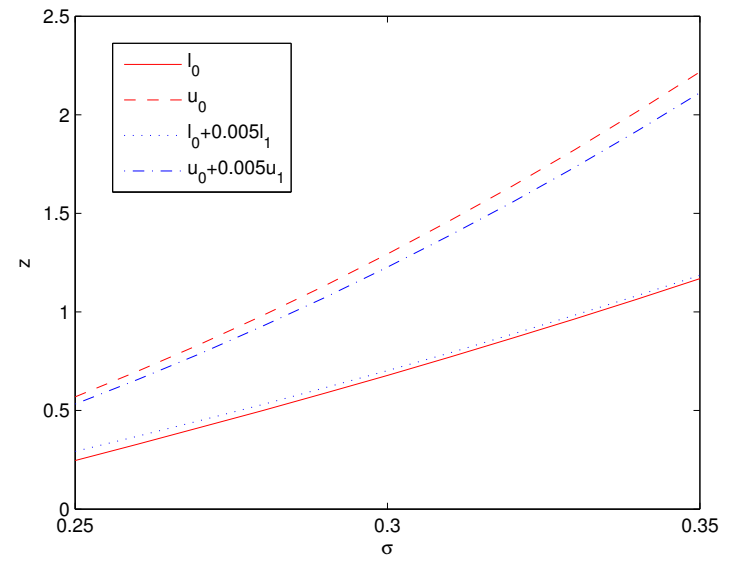

(a) $\delta_{0}=\delta_{1}=0.15$

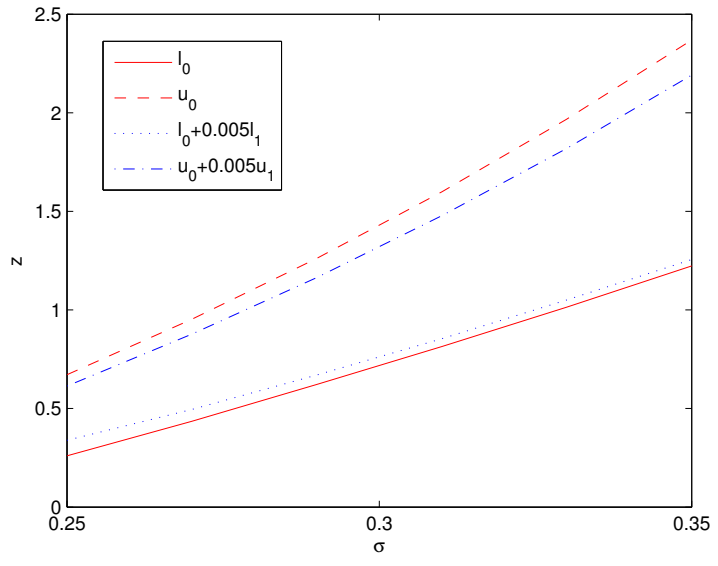

(b) $\delta_{0}=\delta_{1}=0.3$

Figure 7: NT regions as function of volatility $\sigma$.

\section{A Derivation of (47)}

This part is to demonstrate that the expansion for (45) will lead to (47). The expansion for the left hand of the equation is straightforward and therefore omitted. The main challenge of the expansion is the term:

$$
\mathbb{E}_{t, x}\left[\int_{t}^{T} \varphi^{\prime}(s-t) U\left(c_{s}^{*}\right) d s\right] .
$$

We start by introducing the following expansions:

$c^{*}(\cdot)=c_{0}^{*}(\cdot)+\epsilon c_{1}^{*}(\cdot)+o\left(\epsilon^{2}\right), \quad X_{t}=X_{t}^{(0)}+\epsilon X_{t}^{(1)}+o\left(\epsilon^{2}\right), \quad \varphi(\tau)=e^{-\delta_{0} \tau}+\epsilon \Delta(\tau) e^{-\delta_{0} \tau}+o\left(\epsilon^{2}\right)$,

which will be plugged into the equilibrium value function for power utility function:

$$
\begin{aligned}
V(t, x)=\mathbb{E}_{t, x} & {\left[\int_{t}^{T} \varphi(s-t) U\left(c^{*}\left(X_{s}\right)\right) d s\right] } \\
\approx \mathbb{E}_{t, x} & {\left[\int_{t}^{T} \varphi(s-t)\left(\frac{c_{0}^{*}\left(X_{s}^{(0)}\right)^{1-\gamma}}{1-\gamma}+\epsilon c_{0}^{*}\left(X_{s}^{(0)}\right)^{-\gamma} c_{0, x}^{*}\left(X_{s}^{(0)}\right) X_{s}^{(1)}+\epsilon c_{0}^{*}\left(X_{s}^{(0)}\right)^{-\gamma} c_{1}^{*}\left(X_{s}^{(0)}\right)\right) d s\right] } \\
= & \mathbb{E}_{t, x}\left[\int_{t}^{T} e^{-\delta_{0}(s-t)} \frac{c_{0}^{*}\left(X_{s}^{(0)}\right)^{1-\gamma}}{1-\gamma} d s\right]+\epsilon \mathbb{E}_{t, x}\left[\int_{t}^{T} \Delta(s-t) e^{-\delta_{0}(s-t)} \frac{c_{0}^{*}\left(X_{s}^{(0)}\right)^{1-\gamma}}{1-\gamma}+\right. \\
& \left.\quad+e^{-\delta_{0}(s-t)} c_{0}^{*}\left(X_{s}^{(0)}\right)^{-\gamma} c_{0, x}^{*}\left(X_{s}^{(0)}\right) X_{s}^{(1)}+e^{-\delta_{0}(s-t)} c_{0}^{*}\left(X_{s}^{(0)}\right)^{-\gamma} c_{1}^{*}\left(X_{s}^{(0)}\right) d s\right] \\
=: & V_{0}(t, x)+\epsilon V_{1}(t, x) .
\end{aligned}
$$


This leads to the expansion:

$$
\begin{aligned}
& \mathbb{E}_{t, x}\left[\int_{t}^{T} \varphi^{\prime}(s-t) U\left(c_{s}^{*}\right) d s\right] \\
\approx & -\delta_{0} \mathbb{E}_{t, x}\left[\int_{t}^{T} e^{-\delta_{0}(s-t)} \frac{c_{0}^{*}\left(X_{s}^{(0)}\right)^{1-\gamma}}{1-\gamma} d s\right]-\epsilon \delta_{0} \mathbb{E}_{t, x}\left[\int_{t}^{T} \Delta(s-t) e^{-\delta_{0}(s-t)} \frac{c_{0}^{*}\left(X_{s}^{(0)}\right)^{1-\gamma}}{1-\gamma}+\right. \\
& \left.+e^{-\delta_{0}(s-t)} c_{0}^{*}\left(X_{s}^{(0)}\right)^{-\gamma} c_{0, x}^{*}\left(X_{s}^{(0)}\right) X_{s}^{(1)}+e^{-\delta_{0}(s-t)} c_{0}^{*}\left(X_{s}^{(0)}\right)^{-\gamma} c_{1}^{*}\left(X_{s}^{(0)}\right) d s\right]+ \\
& +\epsilon \mathbb{E}_{t, x}\left[\int_{t}^{T} \Delta^{\prime}(s-t) e^{-\delta_{0}(s-t)} \frac{\left[c_{0, s}^{*}\left(X_{s}^{(0)}\right)\right]^{1-\gamma}}{1-\gamma} d s\right] \\
= & -\delta_{0} V_{0}-\epsilon \delta_{0} V_{1}+\epsilon \mathbb{E}_{t, x}\left[\int_{t}^{T} \Delta^{\prime}(s-t) e^{-\delta_{0}(s-t)} \frac{\left[c_{0, s}^{*}\left(X_{s}^{(0)}\right)\right]^{1-\gamma}}{1-\gamma} d s\right],
\end{aligned}
$$

of which the zeroth order term will go into the RHS of the first equation in (47) and the remaining two terms will go into the $V_{1}$ equation.

\section{References}

[1] T. Bjork and A. Murgoci. A general theory of markovian time inconsistent stochastic control problems. Preprint, 2010.

[2] T. Bjork, A. Murgoci, and X. Zhou. Mean-variance portfolio optimization with state-dependent risk aversion. Mathematical Finance, 2012.

[3] B. Bouchard, L. Moreau, and M. H. Soner. Hedging under an expected loss constraint with small transaction costs. arXiv preprint arXiv:1309.4916, 2013.

[4] J. Y. Campbell and L. M. Viceira. Consumption and portfolio decisions when expected returns are time varying. The Quarterly Journal of Economics, 114(2):433-495, 1999.

[5] R. Carmona. Indifference Pricing: Theory and Applications. Princeton Series in Financial Engineering. Princeton University Press, 2008.

[6] G. Chacko and L. M. Viceira. Dynamic consumption and portfolio choice with stochastic volatility in incomplete markets. Review of Financial Studies, 18(4):1369-1402, 2005.

[7] P. Cheridito and M. Kupper. Composition of time-consistent dynamic monetary risk measures in discrete time. International Journal of Theoretical and Applied Finance, 14(01):137-162, 2011.

[8] M. Chernov, A. Ronald Gallant, E. Ghysels, and G. Tauchen. Alternative models for stock price dynamics. Journal of Econometrics, 116(1):225-257, 2003.

[9] V. Coudert and M. Gex. Does risk aversion drive financial crises? testing the predictive power of empirical indicators. Journal of Empirical Finance, 15(2):167-184, 2008.

[10] M. H. Davis and A. R. Norman. Portfolio selection with transaction costs. Mathematics of Operations Research, 15(4):676-713, 1990.

[11] I. Ekeland and A. Lazrak. Being serious about non-commitment: subgame perfect equilibrium in continuous time. arXiv preprint math/0604264, 2006.

[12] I. Ekeland and A. Lazrak. Equilibrium policies when preferences are time inconsistent. arXiv preprint arXiv:0808.3790, 2008. 
[13] I. Ekeland, O. Mbodji, and T. A. Pirvu. Time-consistent portfolio management. SIAM Journal on Financial Mathematics, 3(1):1-32, 2012.

[14] J.-P. Fouque, G. Papanicolaou, R. Sircar, and K. Solna. Multiscale Stochastic Volatility for Equity, Interest Rate, and Credit Derivatives. Cambridge University Press, 2011.

[15] J.-P. Fouque, R. Sircar, and T. Zariphopoulou. Portfolio optimization \& stochastic volatility asymptotics. Preprint, 2013.

[16] C. Harris and D. Laibson. Dynamic choices of hyperbolic consumers. Econometrica, 69(4):935-957, 2001.

[17] C. J. Harris and D. Laibson. Hyperbolic discounting and consumption. In M. Dewatripont, L. P. Hansen, and S. Turnovsky, editors, Advances in Economics and Econometrics: Theory and Applications, Volume 1, pages 258-298. Eighth World Congress, 2002.

[18] Y. Hu, H. Jin, and X. Y. Zhou. Time-inconsistent stochastic linear-quadratic control. SIAM Journal on Control and Optimization, 50(3):1548-1572, 2012.

[19] J. Hull and A. White. The Pricing of Options on Assets with Stochastic Volatilities. Journal of Finance, 42(2):281-300, June 1987.

[20] E. Karni. Risk aversion for state-dependent utility functions: Measurement and applications. International Economic Review, 24(3):pp. 637-647, 1983.

[21] T. C. Koopmans. Stationary ordinal utility and impatience. Econometrica: Journal of the Econometric Society, pages 287-309, 1960.

[22] H. Kraft. Optimal portfolios and Heston's stochastic volatility model: an explicit solution for power utility. Quantitative Finance, 5(3):303-313, 2005.

[23] G. Loewenstein and D. Prelec. Anomalies in intertemporal choice: Evidence and an interpretation. The Quarterly Journal of Economics, 107(2):573-597, 1992.

[24] R. Merton. Continuous-Time Finance. Wiley, 1992.

[25] H. Pham. Continuous-time Stochastic Control and Optimization with Financial Applications. Springer, 2009.

[26] T. A. Pirvu and H. Zhang. Utility indifference pricing: A time consistent approach. Applied Mathematical Finance, 20(4):304-326, 2013.

[27] M. Scheicher. What drives investor risk aversion? daily evidence from the german equity market. BIS Quarterly Review, pages 67-74, 2003.

[28] S. Shreve and H. Soner. Optimal investment and consumption with transaction costs. Ann. Appl. Prob., 4:609692, 1994.

[29] R. H. Strotz. Myopia and inconsistency in dynamic utility maximization. The Review of Economic Studies, pages $165-180,1955$.

[30] N. Tarashev, K. Tsatsaronis, and D. Karampatos. Investors' attitude towards risk: what can we learn from options? BIS Quarterly Review, 6:57-65, 2003.

[31] J. A. Wachter. Portfolio and consumption decisions under mean-reverting returns: An exact solution for complete markets. Journal of financial and quantitative analysis, 37(01):63-91, 2002. 